\title{
Geodätische Linien und ihre Enveloppen auf dreiaxigen Flächen zweiten Grades.
}

\author{
Von \\ A. v. Braunmühl in Munchon. \\ (Mit einer lithogr. Tafel.)
}

\section{Einleitung.}

Nachdem es Jacobi*) im Jahre 1838 gelungen war, das Problem der geodätischen Linien auf dem dreiaxigen Ellipsoide auf Quadraturen zurückzuführen, wodurch dasselbe zugleich für alle Flächen zweiten Grades gelöst erschien, wurde das gleiche Resultat theils geometrisch, theils algebraisch von Joachimsthal**), O.B onn et***), Liou ville†), Chaslest†), Cayley $\dagger+\dagger$ ) und anderen begründet. Die weitere Behandlung der Quadraturen erfolgte jedoch erst 1861 durch Weierstrass*i), welcher auf die Summe der beiden Abel'schen Integrale erster Gattung $(p=2)$, die in der Gleichung der geodätischen Linien auftreten, das Jacobi'sche Unkehrproblem anwandte und dadurch elegante Formeln erhielt, welche die rechtwinkligen Coordinaten eines Punktes der geodätischen Linie auf dem dreiaxigen Ellipsoide durch hyperelliptische $\vartheta$-Functionen von einem variabeln Parameter abbängig machen. Diese Formeln wurden ohne Ableitung mitgetheilt, und Weierstrass bemerkt, dass sich für ein dreiaxiges Ellipsoid, das wenig von einem Sphäroid verschieden ist, rasch convergente Reihen zur Berechnung dieser Coordinaten angeben liessen, die er in einem spätern Aufsatze mitzutheilen verspricht. Meines Wissens ist jedoch die Publication dieser Formeln unterblieben.

*) Am 28. December 1838 theilte Jacobi seine Lösung der Pariser Akademie mit. Vergl. Journal von Crelle, Bd. 19, pag. 309. - Vorlesungen über Dynamik; herausgegeben ron Clebsch, pag. 214.

**) Journal von Crelle, Bd. 26, pag. 155.

***) Journal de l'école polytechnique t. 19, pag. 137.

†) Journal des Mathématiques, t. 9, Serie I, pag. 401-409 und t. 11, pag. 21.

i†) Journal des Mathématiques, t. 11, Serie I, pag. 5, 105, 111.

$+f \dagger$ ) Proceedings of the London mathematical Society, Vol. IV, pag. 191-211.

*†) Monatsberichte der Berliner Akademie, 1861, pag. 986-997. 
Ich stellte mir nun im Folgenden die Aufgabe, die Weierstrass'schen Formeln für eine allgemeine geodätische Linie abzuleiten ( 1 und 2), die dabei auftretenden Constanten in eine für die numerische Berechnung bequemere Form. zu bringen (\$ 3) und überhaupt das Problem soweit zu fördern, dass es für eine Berechnung unmittelbar

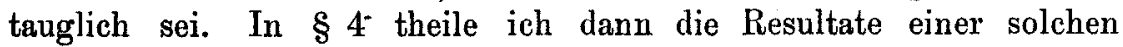
numerischen Rechnung mit, da es mir nicht uninteressant erscheint, die Brauchbarkeit der hyperelliptischen Functionen zu diesem Zwecke einmal an einem Beispiele geprüft zu sehen, zumal da Cayley bereits in zwei grösseren Arbeiten die numerische Bestimmung einer allgemeinen geodätischen Linie auf dem einschaligen Hyperboloide*) und einer Linie durch die Kreispunkte auf dem dreiaxigen Ellipsoide**) mit directer Berechnung der Quadraturen ausführte.

Durch die letztere Arbeit veranlasst theile ich in $\$ 5$ Formeln mit, welche die rechtwinkligen Coordinaten eines Punktes der geodätischen Linie durch die Kreispunkte auf dem Ellipsoide direct von einem Parameter abhängig machen. Eine numerische Rechnung wurde nicht beigefügt, da ausser Cayley auch Rohn***) bereits eine solche bewerkstelligt hat.

Die folgenden Paragraphen besprechen den Hauptgegenstand der vorliegenden Abhandlung. Ich greife nämlich auf einen frühern Aufsatz von mir zurück $\dagger$ ). Anschliessend an die Bemerkung von Ja cobi $\dagger$ ), dass im Allgemeinen jede geodätische Linie nur bis zu einer gewissen Grenze die Eigenschaft hat, kürzeste Linie zu sein, und in Folge dessen ein Büschel geodätischer Linien eine Enveloppe besitzen wird, untersuchte ich damals die Gestalten dieser Enveloppen auf den Rotationsflächen zweiten Grades. Diese Untersuchung wird nun auf das dreiaxige Ellipsoid ausgedehnt. In $\S 6$ werden die Integralgleichungen einer Enveloppe aufgestellt, und Grenzen angegeben, zwischen denen der Berührungspunkt einer beliebigen Linie mit ihrer Enveloppe liegt. In § 7 wird die Lage dieser Berührungspunkte für specielle geodätische Linien discutirt, zu diesem Zwecke ein Hülfssatz bewiesen, die Definition der halben Periode einer geodätischen Linie eingeführt und die Gestalt einer Enveloppe ins Ange gefasst. Zum Schlusse wird das gesammte Resultat in einem Satze vereinigt. $\$ 8$ enthält dann die Folgerungen, die sich aus diesem Satze in Bezug auf die drei Hauptschnitte ergeben, während $\S 9$ die Mittel angiebt, Punkte der Enveloppe durch hyperelliptische Functionen zu berechnen.

*) Proceedings of the London mathematical Society, Vol. IV, p. 371-380.

*) Memoirs of the Royal Astronomical Society, Part. II, Vol. XXXIX, p. 44. ***) Mathematische Modelle, München, Serie I, Nr. b. (Verlag v. L. Brill, Darmstadt.)

†) Diese Annalen Bd. XIV, p. 557-566.

††) Jacobi, Dynamik, pag. 46. 
Die noch folgenden zwei Paragraphen sind dem dreiaxigen Hyperboloid mit zwei Mantelfächen gewidmet. Ihre Veranlassung finden sie in einem vor Jahresfrist erschienenen Aufsatze von Herrn v. Mangold $t^{*}$ ), der mit meinen früheren Untersuchungen in engem Zusammenhang steht. In $\S 10$ wird ein Satz bezüglich der Linien, die durch die Kreispunkte der Fläche gehen, bewiesen, ferner werden die Beziehungen desselben zu der erwähnten Arbeit klar gelegt und endlich in $\$ 11$ die Gleichungen zur Berechnung der Grenzcurve aufgestellt, welche nach Herrn v. Mangoldt diejenigen Punkte der Fläche trennt, die als Ausgangspunkte geodätischer Linien genommen Enveloppen veranlassen oder nicht.

\section{$\S 1$.}

Gleichungen der geodätischen Linien auf dem Ellipsoide.

Als Ausgangspunkt für das Folgende dient uns das erste Integral der Differentialgleichung geodätischer Linien, wie es ron $\mathrm{Jacobi}^{* *}$ ) gegeben worden. Nur in der Bezeichnungsweise schliessen wir uns an Liouville***) an. Es seien nämlich die Gleichungen des Ellipsoids und der dazu confocalen Flächen

$$
\left\{\begin{array}{l}
\frac{x^{2}}{\sigma^{2}}+\frac{y^{2}}{\sigma^{2}-h^{2}}+\frac{z^{2}}{\sigma^{2}-k^{2}}=1, \\
\frac{x^{2}}{\mu^{2}}+\frac{y^{2}}{\mu^{2}-h^{2}}-\frac{z^{2}}{k^{2}-\mu^{2}}=1, \\
\frac{x^{2}}{\nu^{2}}-\frac{y^{2}}{h^{2}-\nu^{2}}-\frac{z^{2}}{k^{2}-\nu^{2}}=1 .
\end{array}\right.
$$

Aus diesen Gleichungen folgt die Bedingung

$$
\infty>\underline{\sigma}>k>\underline{\mu}>h>\underline{v}>0 \text {. }
$$

Lässt man $\mu$ alle möglichen Werthe von $\mu=k$ bis $\mu=h$ annehmen, so bestimmen die beiden ersten dieser Gleichungen das eine System von Krümmungslinien des Ellipsoides, welches ron allen confocalen einschaligen Hyperboloiden ausgeschnitten wird, und man kann somit $\boldsymbol{\mu}$ als Parameter der Krümmunglinien dieses Systems auffassen. Dieselben liegen zwischen den Hauptschnitten $\mu=k$ ( $x y$ Ebene) und $\mu=h$ (xz Ebene). Lässt man $\nu$ von $\nu=0$ bis $\nu=\mathrm{h}$ variiren, so geben die erste und dritte Gleichung das zweite System von Krüm-

*) H. จ. Mangoldt. Ueber diejenigen Punkte auf positiv gekrümmten Flächen, welche die Eigenschaft haben, dass die von ihnen ausgehenden geodätischen Linien nie aufhören, kürzeste Linien zu sein. Journal von CrelleBorchardt. Bd. 91, pag. 23.

**) Dynamik, pag. 214.

***) Liouville. Note III zu Monge's Application de l'Analyse à la Géométrie, pag. 581. 
mungslinien, das somit von den confocalen zweischaligèn Hyperboloiden ausgeschnitten wird und zwischen den Hauptschnitten $\nu=h(x \dot{z}$ Ebene) und $\nu=0$ ( $y z$ Ebene) liegt, $\nu$ kann somit als Parameter des zweiten Systems aufgefasst* werden. Beide Systeme vereinigen sich in den Kreispunkten, für welche $\mu=\nu=h$ ist.

Mit diesen Bezeichnungen wird dann die Gleichung der geodätischen Linien

$$
\left.\int_{k^{2}}^{\mu^{2}} \frac{\left(\sigma^{2}-\mu^{2}\right) d\left(\mu^{2}\right)}{M} \pm \int_{h^{2}}^{v^{2}} \frac{\left(\sigma^{2}-v^{2}\right) d\left(v^{2}\right)}{N}=u^{*}\right)
$$

Hier ist $x$ eine durch Anfangswerthe zu bestimmende Constante,

$$
M^{2}=\left(\sigma^{2}-\mu^{2}\right)\left(k^{2}-\mu^{2}\right)\left(\mu^{2}-\mu^{\prime 2}\right)\left(\mu^{2}-h^{2}\right) \mu^{2},
$$

während $N^{2}$ aus diesem Ausdrucke hervorgeht, indem man $\mu$ mit $\nu$ vertauscht. $\mathrm{Da}$ die Differentialgleichung der geodätischen Linien vom zweiten Grade ist, so ist $\mu^{\prime}$ die Constante dè ersten Integration. Die geometrische Bedeutung dieser Constanten ergiebt sich aus einer Form, in welche zuerst Liouville die Gleichung (2) gebracht hat. ${ }^{* *}$ )

Bezeichnet man nämlich mit $i$ den Winkel, unter welchem eine geodätische Linie eine Krümmungslinie schneidet, so ist diese Gleichung:

$$
\mu^{2} \cos ^{2} i+\nu^{2} \sin ^{2} i=\mu^{\prime 2} \text {. }
$$

Dieselbe wird für $\mu=\mu^{\prime}, i=0$ befriedigt. Somit ist $\mu^{\prime}$ der Parameter jener Krümmungslinie, welche von der geodätischen berührt wird.

Giebt man also ein bestimmtes $\mu^{\prime}$, so sind dadurch alle unendlich vielen geodätischen Linien gegeben, welche die beiden Zweige der dem Werthe von $\mu^{\prime}$ entsprechenden Krämmungslinie berührend um die Fläche oscilliren, während sie in das ausserhalb dieser Zweige befindliche Flächengebiet nicht eintreten. Giebt man in dem inzwischen liegenden Flächentheile einen Punkt $\mu_{0} \nu_{0}$, so gehen durch diesen Punkt immer zwei geodätische Linien, deren Ausgangswinkel durch die Gleichung $\operatorname{tg} i= \pm \sqrt{\frac{\mu_{0}^{2}-\mu^{\prime 2}}{\mu^{\prime 2}-\nu_{0}^{2}}}$, die aus (3) folgt, gegeben ist. Es gehen also durch jeden Punkt der Fläche zqvei geodätische Linien, die die Krümmungslinie $\mu^{\prime}$ berühren. Nur für den Kreispunkt als Ausgangspunkt wird dieser Winkel unbestimmt, da $\mu_{0}=\mu^{\prime}=\nu_{0}=h$ ist; somit kann man durch ihn unendlich viele Linien unter beliebigen Winkeln ziehen, welche bekanntlich insgesammt durch den gegenüberliegenden laufen.

Soll die geodätische Linie reell sein, so muss, wie man aus dem Radikale $M$ sieht, entweder $k>\mu^{\prime}>h$ oder $h>\mu^{\prime}>0$ sein. Im einen Falle berührt sie eine Krummungslinie des ersten, im andern

*) Salmon-Fiedler: Raumgeometrie II. Theil, pag. 162.

**) Journal des Mathémationes, t. 9, Serie I, pag. 403. 
Falle eine des zweiten Systems. Den Uebergang vom ersten zum zweiten System geodätischer Linien (diese Unterscheidung wollen wir in Zukunft beibehalten) bildet damn die Linie durch die Kreispunkte. Wir setzen für das folgende voraus, $\mu^{\prime}$ liege zwischen $k$ und $h$.

\section{$\S 2$.}

Einführung der hyperelliptischen $\vartheta$-Functionen.

Löst man die Gleichungen (1) nach $x, y, z$ auf, so erhält man die rechtwinkligen Coordinaten eines. Punktes auf dem Ellipsoide durch elliptische Coordinaten $\mu$ und $\nu$ ausgedrückt, in der Form:

$$
\left\{\begin{array}{l}
x=\frac{\sigma}{h k} \cdot \mu \cdot \nu, \\
y=\frac{1}{h} \sqrt{\frac{\sigma^{2}-h^{2}}{k^{2}-h^{2}}} \sqrt{\left(\mu^{2}-h^{2}\right)\left(h^{2}-\nu^{2}\right)}, \\
z=\frac{1}{h} \sqrt{\frac{\sigma^{2}-h^{2}}{k^{2}-h^{2}}} \sqrt{\left(k^{2}-\mu^{2}\right)\left(k^{2}-\nu^{2}\right)} .
\end{array}\right.
$$

Bringt mau danit die durch die Gleichung (2) zwischen $\mu$ und $\nu$ gegebene Relation in Verbindung, so sind dies die Coordinaten eines Punktes der geodätischen Linie. Nimmt man nun mit Weierstrass ${ }^{* *}$ ) zu der Gleichung (2) noch eine ähnlich gebildete

$$
\int_{k^{2}}^{\mu^{2}} \frac{d\left(\mu^{2}\right)}{M} \pm \int_{h^{2}}^{v^{2}} \frac{d\left(\nu^{2}\right)}{N}=u
$$

hinzu, wo $u=-\int \frac{d s}{\left(\sigma^{2}-\mu^{2}\right)\left(\sigma^{2}-v^{2}\right)}$ und $d s$ das Bogendifferential der geodätischen Linie bedeutet, so ist $u$ für die Punkte der geodätischen Linie variabel, und man kann auf (2) und (5) die Jacubi'sche Umkehr anwenden. Um die ferneren Entwicklungen mit den von Rosen. hain***) gegebenen Formeln direct in Einklang zu bringen, wenden wir auf diese beiden Gleichungen die Substitution an

$$
\begin{aligned}
\mu^{2}=\nu^{2}=k^{2} \frac{1-\lambda^{2} x}{1-\rho^{2} x}, \quad \varkappa^{2} & =\frac{\sigma^{2}-h^{2}}{k^{2}-h^{2}} \cdot \frac{k^{2}-\mu^{\prime 2}}{\sigma^{2}-\mu^{\prime 2}} ; \quad \lambda^{2}=\frac{k^{2}-\mu^{\prime 2}}{\sigma^{2}-\mu^{2} \cdot \frac{a^{2}}{\hbar^{2}},} \\
\rho^{2} & =\frac{k^{2}-\mu^{\prime 2}}{\sigma^{2}-\mu^{\prime 2}},
\end{aligned}
$$

wodurch die Gleichungen (2) und (5) übergehen in:

*) Liouville. Note III zu Monge's Application etc. pag. 581.

**) Weierstrass a. a. O. pag. 994.

***) Rosenhain: Mémoirs des savants étrangers, t. 11. 
(6)

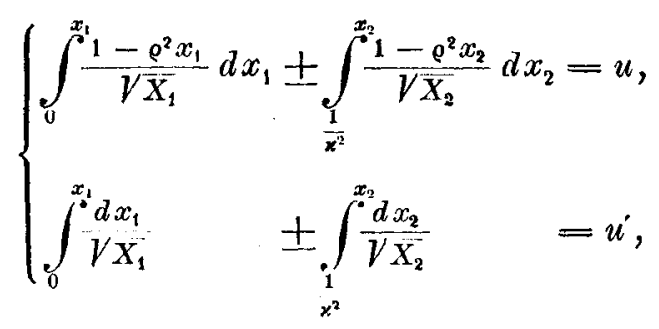

wo $X=x(1-x)\left(1-x^{2} x\right)\left(1-\lambda^{2} x\right)\left(1-\rho^{2} x\right)$ ist, während die Gleichungen (4) die Form annehmen:

$$
\left\{\begin{array}{l}
x^{2}=a_{1} \frac{\left(1-\lambda^{2} x_{1}\right)\left(1-\lambda^{2} x_{2}\right)}{\left(1-e^{2} x_{1}\right)\left(1-\rho^{2} x_{2}\right)} \\
y^{2}=-a_{2} \frac{\left(1-x^{2} x_{1}\right)\left(1-x^{2} x_{2}\right)}{\left(1-e^{2} x_{1}\right)\left(1-e^{2} x_{2}\right)} \\
z^{2}=\quad a_{3} \frac{x_{1} x_{2}}{\left(1-e^{2} x_{1}\right)\left(1-\rho^{2} x_{2}\right)}
\end{array}\right.
$$

$a_{1}, a_{2}, a_{3}$ sind leicht zu bestimmende Constante.

Schreibt man jetzt die ersten 5 Formeln der von Rosenhain a. a. O. pag. 422 gegebenen Formeltafel in die bequemere Rie mann'sche Bezeichnungsweise um, so hat man:

$$
\begin{aligned}
& \text { (a) } \quad x \lambda \rho x_{1} x_{2}=\frac{\vartheta^{2}\left(\begin{array}{ll}
1 & 0 \\
1 & 1
\end{array}\right)(v \mid w)}{\vartheta^{2}\left(\begin{array}{ll}
0 & 0 \\
1 & 1
\end{array}\right)(v \mid w)} \text {, } \\
& \text { b) }-\frac{x \lambda \rho}{x_{1} \lambda_{1} \varrho_{1}}\left(1-x_{1}\right)\left(1-x_{2}\right)=\frac{\vartheta^{2}\left(\begin{array}{ll}
1 & 0 \\
0 & 1
\end{array}\right)(v \mid w)}{\vartheta^{2}\left(\begin{array}{ll}
0 & 0 \\
1 & 1
\end{array}\right)(v \mid w)} \text {, }
\end{aligned}
$$

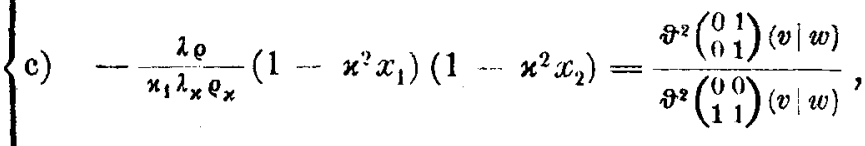

$$
\begin{aligned}
& \text { d) } \quad \frac{x \rho}{\lambda_{1} \varrho_{\lambda} \lambda_{x}}\left(1-\lambda^{2} x_{1}\right)\left(1-\lambda^{2} x_{2}\right)=\frac{\vartheta^{2}\left(\begin{array}{ll}
0 & 1 \\
0 & 0
\end{array}\right)(v \mid w)}{\vartheta^{2}\left(\begin{array}{ll}
0 & 0 \\
1 & 1
\end{array}\right)(v \mid w)} \text {, } \\
& \text { e) } \frac{x^{2}}{\varrho_{1} \varrho_{x} \rho_{\lambda}}\left(1-\varrho^{2} x_{1}\right)\left(1-\varrho^{2} x_{2}\right)=\frac{\vartheta^{2}\left(\begin{array}{ll}
0 & 0 \\
0 & 0
\end{array}\right)(v \mid w)}{\mathfrak{\vartheta}^{2}\left(\begin{array}{ll}
0 & 0 \\
1 & 1
\end{array}\right) \overline{(v \mid w)}} \text {, }
\end{aligned}
$$

und wenn man diese symmetrischen Functionen von $x_{1}, x_{2}$ in die Gleichungen (7) einführt, so folgt: 


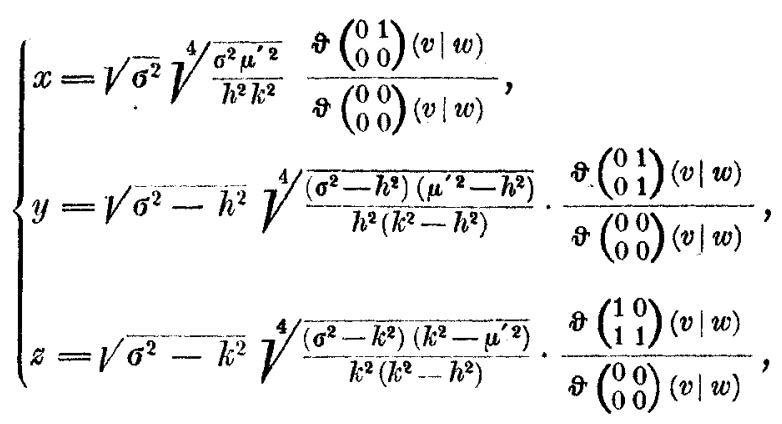

welche Gleichungen bis auf die Bezeichnung mit denen von Weierstrass a. a. O. erhaltenen übereinstimmen. Die $\vartheta$-Functionen sind hier definirt durch die Gleichung:

$$
\begin{aligned}
\left.\left.\vartheta\left(\begin{array}{ll}
h_{1} & h_{2} \\
g_{1} & g_{2}
\end{array}\right)=\sum_{-\infty}^{+\infty} \sum_{-\infty}^{+\infty} p^{\left(m+\frac{h_{1}}{2}\right)} \cdot q^{\left(n+\frac{h_{2}}{2}\right)} \cdot e^{2\left(m+\frac{h_{1}}{2}\right)\left(n+\frac{h_{2}}{2}\right) a_{1,2}} \cdot \cos \Omega_{m, n}\right)^{*}\right) \\
\Omega_{m, n}=2\left(m+\frac{h_{1}}{2}\right)\left(v-\frac{g_{1}}{2}\right) \pi+2\left(n+\frac{h_{2}}{2}\right)\left(w-\frac{g_{2}}{2}\right) \pi,
\end{aligned}
$$

während die seit Richelot gebräuchlichen Bezeichnungen $\lambda_{1}{ }^{2}=1-\lambda^{2}$, $\lambda_{x}{ }^{2}=x^{2}-\lambda^{2}$ etc. verwendet sind. Sollen nun die Gleichungen (9) die Coordinaten eines Punktes der geodätischen Linie darstellen, so müssen $v$ und $w$ linear abhängig sein von der Constanten $u^{\prime}$ und der Variabeln $u$; man muss also haben:

$$
\left\{\begin{array}{c}
v=\beta_{1,1} u+\beta_{1,2} u^{\prime}, \\
w=\beta_{2,1} u+\beta_{2,2} u^{\prime} .
\end{array}\right.
$$

Die hier auftretenden Constanten sowie die Constanten $p, q, e \pm 2 a_{1,2}$ der $\vartheta$-Functionen müssen nun noch passend bestimmt werden. Ist dieses geschehen, so sind $v$ und $v$ nur mehr von der einen Variabeln $u$ abhängig, und wenn $u$ von $-\infty$ bis $+\infty$ läuft, so geben die Gleichungen (9) sämmtliche Punkte der geodätischen Linie.

\section{$\S 3$.}

\section{Constantenbestimmung.}

Die vier Constanten $\beta_{1,1}, \beta_{1,2}, \beta_{2,1}, \beta_{2,2}$ sind von Weierstrass durch die Periodicitätsmoduln der Integrale, die in den Gleichungen (2) und (5) auftreten, ausgedrückt. Diese Bestimmung kann jedoch durch eine für numerische Rechnung zweckmässigere ersetzt werden. Die Berechnung der erwähnten Moduln auf dem Wege der Quadratur

*) Vergl. Thomae: Sammlung von Formeln, welche bei Anwendung ron elliptischen und Rosenhain'schen Functionen gebraucht werden, pag. 9, Formel 101 a und Weierstrass a. a. O. pag. 994 und 995. 
erfordert nämlich schon in günstigen Fällen eine grosse Gliederzahl. Wir drücken sie daher durch Quotienten von $\boldsymbol{\vartheta}$-Functionen mit verschwindenden Argumenten aus, indem wir uns eines in dem Werke von Clebsch und Gordan: "Theorie der Abel'schen Functionen" pag. 331 ausgesprochenen Gedankens bedienen.

Aus den Gleichungen (6) erhält man nämlich durch Auflösung nach $d x_{1}$ und $d x_{2}$

$$
\begin{aligned}
& d x_{1}=\frac{V \overline{X_{1}}}{\rho^{2}\left(x_{2}-x_{1}\right)} d u-\frac{V \overline{X_{1}}\left(1-\rho^{2} x_{2}\right)}{\rho^{2}\left(x_{2}-x_{1}\right)} d u^{\prime}, \\
& d x_{2}=\frac{V \overline{X_{2}}}{\rho^{2}\left(x_{1}-x_{2}\right)} d u-\frac{V X_{2}\left(1-\rho^{2} x_{1}\right)}{\rho^{2}\left(x_{1}-x_{2}\right)} d u^{\prime} .
\end{aligned}
$$

Differentiirt man jetzt die Gleichungen a) und c) in (8), führt für die auftretenden Differentiale $d x_{1}, d x_{2}$ die obigen Werthe ein und setzt dann $x_{1}=0, x_{2}=\frac{1}{x^{2}}$, d. h. $v=w=0$, so gewinnt man die Formeln:

$$
\begin{aligned}
& \frac{\vartheta^{\prime}\left(\begin{array}{ll}
1 & 0 \\
1 & 1
\end{array}\right)_{v}}{\vartheta\left(\begin{array}{ll}
0 & 0 \\
1 & 1
\end{array}\right)} d v+\frac{\vartheta^{\prime}\left(\begin{array}{ll}
1 & 0 \\
1 & 1
\end{array}\right) w}{v\left(\begin{array}{ll}
0 & 0 \\
1 & 1
\end{array}\right)} d w=\frac{1}{2} \frac{x}{\rho} \sqrt{\frac{x \lambda}{\rho}} d u-\frac{1}{2} \frac{\varrho_{x}^{2}}{\rho} \sqrt{\frac{\lambda}{x \cdot \rho}} d u^{\prime}
\end{aligned}
$$

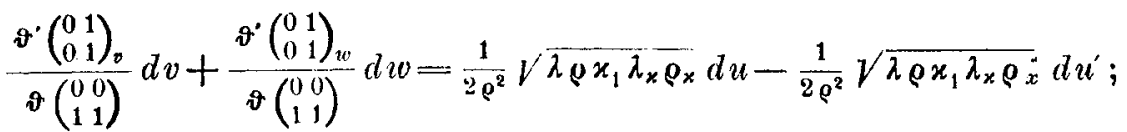

bierin ist $\vartheta^{\prime}\left(\begin{array}{ll}h_{1} & h_{2} \\ g_{1} & g_{2}\end{array}\right)$ der Werth, welchen die nach $v$ gebildete $\mathrm{Ab}$ geleitete ron $\vartheta\left(\begin{array}{ll}h_{1} & h_{2} \\ g_{1} & g_{2}\end{array}\right)(v \mid w)$ annimmt, wenn man $v=w=0$ setzt, während $\vartheta\left(\begin{array}{ll}h_{1} & h_{2} \\ g_{1} & g_{2}\end{array}\right)$ für $\vartheta\left(\begin{array}{ll}h_{1} & h_{2} \\ g_{1} & g_{2}\end{array}\right)(0 \mid 0)$ gesetzt wurde.

Löst man diese Gleichungen nach $d v$ und $d w$ auf und vergleicht die Coefficienten von $d u$, $d u^{\prime}$ mit denen der Differentialquotienten der Gleichungen (10), so erhält man:

$$
\left\{\begin{array}{l}
\beta_{1,1}=\frac{1}{\Delta} \sqrt{\frac{\lambda}{\varrho^{3}}}\left\{\sqrt{x_{1} \lambda_{x} \varrho_{x}} \vartheta^{\prime}\left(\begin{array}{ll}
1 & 0 \\
1 & 1
\end{array}\right)_{w}-\sqrt{x^{3}} \vartheta^{\prime}\left(\begin{array}{ll}
0 & 1 \\
0 & 1
\end{array}\right)_{w}\right\}, \\
\beta_{1,2}=\frac{1}{\Delta} \sqrt{\frac{\varrho_{x}^{2}}{\rho^{3}}}\left\{\sqrt{\frac{\varrho^{3}}{x}} \vartheta^{\prime}\left(\begin{array}{ll}
0 & 1 \\
0 & 1
\end{array}\right)_{w}-\sqrt{x_{1} \lambda_{x}} \vartheta^{\prime}\left(\begin{array}{ll}
1 & 0 \\
1 & 1
\end{array}\right)_{w}\right\} \\
\beta_{2,1}=\frac{1}{\Delta} \sqrt{\frac{\lambda}{\varrho^{3}}}\left\{\sqrt{x^{3}} \vartheta^{\prime}\left(\begin{array}{ll}
0 & 1 \\
0 & 1
\end{array}\right)_{v}-\sqrt{x_{1} \lambda_{x} \varrho_{x}} \vartheta^{\prime}\left(\begin{array}{ll}
1 & 0 \\
1 & 1
\end{array}\right)_{v}\right\} \\
\beta_{2,2}=\frac{1}{\Delta} \sqrt{\frac{\varrho_{x} \lambda}{\rho^{3}}}\left\{\sqrt{x_{1} \lambda_{x}} \vartheta^{\prime}\left(\begin{array}{ll}
1 & 0 \\
1 & 1
\end{array}\right)_{v}-\sqrt{\frac{\varrho_{x}^{3}}{x}} \vartheta^{\prime}\left(\begin{array}{ll}
0 & 1 \\
0 & 1
\end{array}\right)_{v}\right\}
\end{array} .\right.
$$


$\Delta$ hat ilen Werth $2 \frac{\vartheta^{\prime}\left(\begin{array}{ll}1 & 0 \\ 1 & 1\end{array}\right)_{w} \vartheta^{\prime}\left(\begin{array}{ll}0 & 1 \\ 0 & 1\end{array}\right)_{v}-\vartheta^{\prime}\left(\begin{array}{ll}1 & 0 \\ 1 & 1\end{array}\right)_{v} \vartheta^{\prime}\left(\begin{array}{ll}0 & 1 \\ 0 & 1\end{array}\right)_{w}}{\vartheta\left(\begin{array}{ll}0 & 0 \\ 1 & 1\end{array}\right)} \cdot$ Dieser Werth lässt sich noch für die Rechnung bequemer gestalten, wenn man die bekannte Formel

$$
\begin{gathered}
\vartheta^{\prime}\left(\begin{array}{ll}
0 & 1 \\
0 & 1
\end{array}\right)_{v} \vartheta^{\prime}\left(\begin{array}{ll}
1 & 0 \\
1 & 1
\end{array}\right)_{w}-\vartheta^{\prime}\left(\begin{array}{ll}
0 & 1 \\
0 & 1
\end{array}\right)_{w} \vartheta^{\prime}\left(\begin{array}{ll}
1 & 0 \\
1 & 1
\end{array}\right)_{v} \\
\left.=-\pi^{2} \vartheta\left(\begin{array}{ll}
0 & 0 \\
0 & 0
\end{array}\right) \vartheta\left(\begin{array}{ll}
0 & 0 \\
1 & 1
\end{array}\right) \vartheta\left(\begin{array}{ll}
0 & 1 \\
0 & 0
\end{array}\right) \vartheta\left(\begin{array}{ll}
1 & 0 \\
0 & 1
\end{array}\right) *\right) \text { benütet }
\end{gathered}
$$

und $\vartheta\left(\begin{array}{ll}0 & 1 \\ 0 & 0\end{array}\right), \vartheta\left(\begin{array}{ll}1 & 0 \\ 0 & 1\end{array}\right)$ mit den von Rosenhain a. a. 0. pay. 418 gegebenen Formeln durch $x, \lambda, \varrho$ ausdrückt. Man erhält dann nämlich: $\Delta=-2 \pi^{2} \vartheta\left(\begin{array}{ll}0 & 0 \\ 0 & 0\end{array}\right)^{3} \frac{\varrho}{\lambda_{1} \varrho_{x}} \sqrt{\frac{{x_{1} \rho_{1} \lambda_{x} \rho_{\lambda}}_{\lambda}}{\lambda}}$. Um aus diesen Formeln die Constanten $\beta$ zu berechnen, ist, wie man sieht, nur die Kenntniss von $p, q, e^{ \pm a_{12}}$ nöthig.**) Diese kann man auf ähnliche Weise finden, wie man in der Theorie der elliptischen Functionen aus dem Modul $x$ den Werth von $q$ bestimmt. Aus den 9 Gleichungen, die Rosenhain pag. 418 seiner schon öfters erwähnten. Abhandlung angiebt, und welche die Beziehungen zwischen den Moduln $x, \lambda, \varrho$ und den $\boldsymbol{\vartheta}$ Quotienten mit verschwindenden Argumenten bestimmen, wählen wir diejenigen drei aus, in welche $p, q$ und $e^{ \pm 2 c_{12}}$ nur in ganzzahligen Potenzen eingehen. Zieht man aus diesen die positiven vierten Wurzeln und setzt sie in unsere Bezeichnungsweise um, so lauten dieselben:

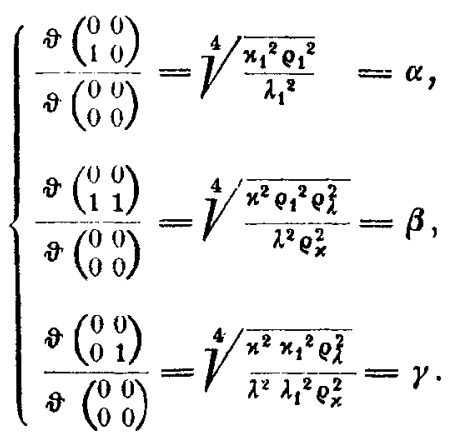

*) Thomae a. a. 0., p. 17, Formel 146, und Rosenhain a. a. O., p. 433.

**) Die von Thomae a. a. $0 .$, p. 12 angegebenen und p. 19 entwickelten Formelu zur Berechnung von $p, q, e^{ \pm a_{12}}$ konnten nicht benützt werden, da sie mir nicht richtig zu sein scheinen. In den dort angegebenen Anfangsgliedern der จ-Reihen müssen nämlich statt der Glieder

$$
2 r p^{3}=2 p^{4} q\left(e^{2 a_{12}}+e^{-2 a_{12}}\right) \quad \text { und } 2 r q^{3}=2 p q^{4}\left(e^{2 a_{12}}+e^{-2 a_{12}}\right)
$$

die Glieder

$$
2 p^{4} q\left(e^{4 a_{12}}+e^{-4 a_{12}}\right) \quad \text { und } \quad 2 p q^{4}\left(e^{4 a_{12}}+e^{-4 a_{12}}\right)
$$

stehen. Hierdurch werden aber die F'ormeln pag. 17 wesentlich geändert. 
A. v. Braungüht.

Setzt man in den $\vartheta$-Functionen $e^{2 a_{12}}+e^{-2 a_{12}}=r$, so sind die Anfangsglieder derselben, welche für uusere späteren $\mathrm{Z}$ wecke genügen:

$$
\left\{\begin{array}{l}
\vartheta\left(\begin{array}{l}
00 \\
00
\end{array}\right)=1+2 p+2 q+2 p q r+2 p^{4}+2 q^{4}+2\left(p q^{4}+p^{4} q\right)\left(r^{2}-2\right)+\cdots \\
\vartheta\left(\begin{array}{l}
00 \\
10
\end{array}\right)=1-2 p+2 q-2 p q r+2 p^{4}+2 q^{4}-2\left(p q^{4}-p^{4} q\right)\left(r^{2}-2\right)+\cdots \\
\vartheta\left(\begin{array}{l}
00 \\
11
\end{array}\right)=1-2 p-2 q+2 p q r+2 p^{4}+2 q^{4}-2\left(p q^{4}+p^{4} q\right)\left(r^{2}-2\right)+\cdots \\
\vartheta\left(\begin{array}{l}
00 \\
01
\end{array}\right)=1+2 p-2 q-2 p q r+2 p^{4}+2 q^{4}+2\left(p q^{4}-p^{4} q\right)\left(r^{2}-2\right)+\cdots
\end{array}\right.
$$

Bildet man hieraus und aus (12) mit Thomae die Gleichungen:

$$
\left\{\begin{array}{l}
p_{u}=\frac{1}{2} \frac{1-\alpha-\beta+\gamma}{1+\alpha+\beta+\gamma}=p+p q^{4}\left(r^{2}-2\right), \\
q_{0}=\frac{1}{2} \frac{1+\alpha-\beta-\gamma}{1+\alpha+\beta+\gamma}=q+q p^{4}\left(r^{2}-2\right), \\
r_{0}=\frac{1}{2} \frac{1-\alpha+\beta-\gamma}{1+\alpha+\beta+\gamma}=p q r,
\end{array}\right.
$$

so folgt, wemn man noch $q^{1}-p^{4}$ vernachlïssigt*), leicht:

$$
\left\{\begin{array}{l}
p=\frac{p_{0} q_{0}}{q_{0}^{2}+p_{0}^{2} r_{0}^{2}} \\
q==\frac{q_{0}{ }^{3}}{q_{0}^{2}+p_{0}{ }^{2} r_{0}{ }^{2}} \\
r=\frac{r_{0}}{p q} .
\end{array}\right.
$$

Bestimmt man hieraus den Werth von $r$, so ergiebt sich $a_{12}$ vermöge der Gleichung

$$
e^{ \pm 2 a_{12}}=\frac{r \pm \sqrt{r^{2}-4}}{2}
$$

Welcher vou den hieraus folgenden reciproken Werthen vou $a_{12}$ genommen werden muss, kann aus dem Quotienten $\frac{\vartheta\left(\begin{array}{ll}1 & 1 \\ 0 & 0\end{array}\right)(v \mid v)}{\vartheta\left(\begin{array}{ll}1 & 1 \\ 1 & 1\end{array}\right)(v \mid w)}$, der einerseits nahezu gleich $-\frac{e^{\frac{a_{12}}{2}}+e^{-\frac{a_{12}}{2}}}{e^{\frac{a_{12}}{2}}-e^{-\frac{a_{12}}{2}}}$, andererseits gleich $\sqrt[4]{\frac{x^{2} \lambda_{1}^{2} \rho_{1}^{2}}{\rho_{x}^{2} \rho_{2}^{2}}}$ ist, entrnommen werden **).

*) Ueber die Guiltigkeitsgrenzen dieser Formeln vergl. §4.

**) Vergl. Thomae a. a. O. pag. 13. 


\section{§4.}

Numerische Berechnung einer geodätischen Linie.

Die in Gleichung (2) auftretende Constante $u^{\prime}$ kann dadurch bestimmt werden, dass man die geodütische Linie durch einen bestimmten Punkt der $x y$-Ebene gehen lässt. Seine elliptischen Coordinaten sind dann $\mu=k, \nu=\nu_{0}$. Dann ist

$$
u^{\prime}=\int_{h^{2}}^{v_{0}{ }^{2}{ }^{2}} \frac{\left(\sigma^{2}-\nu^{2}\right) d\left(\nu^{2}\right)}{N} .
$$

Diese Constante verschwindet, wenn man $\nu_{0}$ mit $h$, d. h. den Anfangspunkt der geodätischen Linie mit dem einen Endpunkte der grossen Axe zusammenfallen lässt. Hierdurch wird die Gestalt der Linie nicht particularisirt, während die Rechnung sich vereinfacht, indem $v=\beta_{1,1} u, w=\beta_{2,1} u$ wird; deshalb sei für das Folgende diese Voraussetzung gemacht. In den Gleichungen (6) ist dann das untere Keichen zu nehmen, und $\mu^{\prime}$ liegt zwischen $k$ und $h$. Für die numerische Rechnung bediente ich mich des Axenverhältnisses

$$
\sigma^{2}:\left(\sigma^{2}-h^{2}\right):\left(\sigma^{2}-k^{2}\right)=\sqrt{3}: \sqrt{2}: \sqrt{1} \text {. }
$$

Was die in $\$ 2$ gebrauchte Substitution anlangt, so sind für ihre Wahl drei Gesichtspunkte massgebend: einmal muss man trachten, die Werthe $p, q, r$ und namentlich den letzten, welcher eine rasche Convergenz der $\vartheta$-Reihe am meisten beeinträchtigt, möglichst klein zu machen, daun sind diejenigen $\vartheta$-Functionen zu vermeiden, welche vermöge ihrer Bildung mehr Glieder beanspruchen, das sind die Reihen mit der Charakteristik $\left(\begin{array}{ll}1 & 1 \\ g_{1} & g_{2}\end{array}\right)$, und endlich muss man suchen, für $u$ und $u$ reelle Werthe zu erhalten. Diese drei Bedingungen erfüllt die oben gewählte Substitution für Werthe von $\mu^{\prime 2}$ zwischen $\mu^{\prime 2}=\frac{1}{2}\left(k^{2}+h^{2}\right)$ und $\mu^{\prime 2}=k^{2}$, während man für Werthe zwischen den Grenzen $\mu^{\prime 2}=h^{2}$ und $\mu^{\prime 2}=\frac{1}{2}\left(k^{2}+h^{2}\right)$ besser

$$
\mu^{2}=\nu^{2}=\frac{h^{2} \mu^{\prime 2}}{\mu^{\prime 2}-\left(\mu^{\prime 2}-h^{2}\right) x}
$$

wählen wird. Begnügt man sich dann mit einer Genauigkeit von 4 Decimalen, so genügt die Gliederzahl in den Gleichungen (13) und die für die Rechnung nöthigen Anfangsglieder der $\vartheta$-Functionen und ihrer Differentialquotienten sind folgende: 
$\boldsymbol{\vartheta}\left(\begin{array}{l}01 \\ 00\end{array}\right)(v \mid w)=2\left\{q^{\frac{1}{4}} \cos w \pi+q^{\frac{9}{4}} \cos 3 w \pi\right.$ $+p q^{\frac{1}{4}}\left(e^{-a_{1 \cdot 2}} \cos (2 v-w) \pi+e^{a_{1,2}} \cos (2 v+w) \pi\right)$

$\left.+p q^{\frac{9}{4}}\left(e^{-3 a_{1,2}} \cos (2 v-3 w) \pi+e^{3 a_{1 \cdot 2}} \cos (2 v+3 w) \pi\right)+\cdots\right\}$, $\vartheta\left(\begin{array}{ll}0 & 1 \\ 0 & 1\end{array}\right)(v \mid w)=2\left\{q^{\frac{1}{4}} \sin w \pi-q^{\frac{9}{4}} \sin 3 w \pi\right.$ $-p q^{\frac{1}{4}}\left(e^{-a_{1,2}} \sin (2 v-w) \pi-e^{a_{1,2}} \cos (2 v+w) \pi\right)$

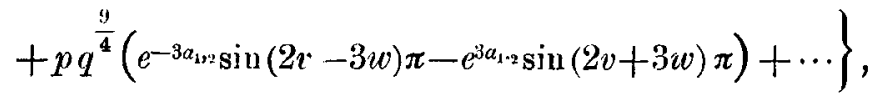
$\vartheta\left(\begin{array}{ll}1 & 0 \\ 1 & 1\end{array}\right)(v \mid w)=2\left\{p^{\frac{1}{4}} \sin v \pi-p^{\frac{9}{4}} \sin 3 v \pi\right.$ $-v^{\frac{1}{4}} q\left(e^{-a_{1,2}} \sin (v-2 w) \pi+e^{a_{1,2}} \sin (v+2 w) \pi\right)$ $\left.+p^{\frac{9}{4}} q\left(e^{-3 a_{1,2}} \sin (3 v-2 w) \pi+e^{3 a_{1,2}} \sin (3 v+2 w) \pi\right)+\cdots\right\}$, $\vartheta\left(\begin{array}{l}00 \\ 00\end{array}\right)(v \mid w)=1+2 p \cos 2 v \pi+2 q \cos 2 w \pi$ $+2 p q\left(e^{\left.-2 n_{1,2} \cos 2(v-w) \pi+e^{2 a_{1,2}} \cos 2(v+w) \pi\right)+\cdots ;}\right.$

(18)

$$
\left\{\begin{array}{l}
\vartheta^{\prime}\left(\begin{array}{l}
10 \\
1
\end{array}\right)_{v}=2 \pi\left\{p^{\frac{1}{4}}-3 p^{\frac{9}{4}}-p^{\frac{1}{4}} q\left(e^{-a_{1,2}}+e^{a_{1,2}}\right)+3 p^{\frac{9}{4}} q\left(e^{-3 a_{1,2}}+e^{3 a_{1,2}}\right)+\cdots\right\} \\
\vartheta^{\prime}\left(\begin{array}{l}
10 \\
11
\end{array}\right)_{w}=4 \pi\left\{p^{\frac{1}{4}} q\left(e^{-a_{1,2}}-e^{a_{1,2}}\right)+p^{\frac{9}{4}} q\left(e^{-3 a_{1,2}}-e^{3 a_{1,2}}\right)+\cdots\right\}, \\
\vartheta^{\prime}\left(\begin{array}{l}
01 \\
01
\end{array}\right)_{v}=4 \pi\left\{-p q^{\frac{1}{4}}\left(e^{-a_{1,2}}-e^{a_{1,2}}\right)+p q^{\frac{1}{4}}\left(e^{-3 a_{1,2}}-e^{3 a_{1,2}}\right)-\cdots\right\}, \\
\vartheta^{\prime}\left(\begin{array}{l}
01 \\
01
\end{array}\right)_{w}=2 \pi\left\{q^{\frac{1}{4}}-3 q^{\frac{9}{4}}+p q^{\frac{1}{4}}\left(e^{-a_{1,2}}+e^{a_{1,2}}\right)-3 p q^{\frac{9}{4}}\left(e^{-3 a_{1,2}}+e^{3 a_{1,2}}+\cdots\right\}\right.
\end{array}\right.
$$

Wählt man endlich die specielle geodätische Linie, die für

$$
\mu^{\prime 2}=\frac{1}{2}\left(k^{2}+h^{2}\right)
$$

resultirt, so ergeben sich folgende Zahlenwerthe für die Constanten:

$$
\begin{array}{lll}
\sigma^{2}=1,7320 \cdot 5, & \mu^{\prime 2}=0,5249 \cdot 4, & x^{2}=0,5857 \cdot 8 \\
k^{2}=0,7320 \cdot 5, & & \lambda^{2}=0,4059 \cdot 4, \\
h^{2}=0,3178 \cdot 3, & & \varrho^{2}=0,1715 \cdot 7 .
\end{array}
$$


Die Formeln (12) und (13) ergeben dann die Werthe

$$
\begin{aligned}
& \alpha=0,8717 \cdot 8, \\
& \beta=0,9068 \cdot 8, \\
& \gamma=0,8686 \cdot 4,
\end{aligned}
$$

und diese liefern mittelst der Formeln (14) und (15)

$$
\begin{array}{ll}
p_{0}=0,0123 \cdot 3, & p=0,0123 \cdot 2, \\
q_{0}=0,0131 \cdot 9, & q=0,0131 \cdot 9, \\
r_{0}=0,0228 \cdot 1, & r=140,3160 .
\end{array}
$$

Die formel (1.6) lässt hieraus $\left.\log e^{+2 a_{12}}=7,85291^{*}\right)$ und $\log e^{-2 a_{12}}$ $=2,14709$ folgen.

Mit diesen Werthen erhält man aus den Gleichungen (18)

$$
\begin{array}{r}
\log \vartheta^{\prime}\left(\begin{array}{ll}
1 & 0 \\
1 & 1
\end{array}\right)_{v}=0,25147, \\
\log \vartheta^{\prime}\left(\begin{array}{ll}
1 & 0 \\
1 & 1
\end{array}\right)_{w}=\dot{9}, 80319, \\
\log -\vartheta^{\prime}\left(\begin{array}{ll}
0 & 1 \\
0 & 1
\end{array}\right)_{v}=\dot{9}, 77984, \\
\log \vartheta^{\prime}\left(\begin{array}{ll}
0 & 1 \\
0 & 1
\end{array}\right)_{w}=0,38357, \\
\log (-\Delta)=0,97533 .
\end{array}
$$

Hieraus fliessen mittelst der Gleichungen (11) die Werthe

$$
\begin{aligned}
& \beta_{1,1}=0,4288 \cdot 3, \\
& \beta_{2,1}=0,3648 \cdot 3,
\end{aligned}
$$

Die Werthe von $\beta_{1,2}$ und $\beta_{2,2}$ können im vorliegenden Falle entbehrt werden, da $u^{\prime}=0$ ist. Die Logarithmen der in den Formeln (9) auftretenden Constanten sind dann der Reihe nach:

$$
\begin{aligned}
& \log \sqrt{\sigma^{2}} \sqrt[4]{\frac{\sigma^{2} \mu^{\prime}-\overline{2}}{k^{2} h^{2}}}=0,26726, \\
& \log \sqrt{\sigma^{2}-h^{2}} \sqrt[4]{ }^{\frac{\left(\sigma^{2}-h^{2}\right)\left(\mu^{\prime} 2-h^{2}\right)}{h^{2}\left(k^{2}-h^{2}\right)}}=0,16208, \\
& \log \sqrt{\sigma^{2}-k^{2}} \sqrt[4]{ }^{\frac{\left(\sigma^{2}-k^{2}\right)\left(k^{2}-\mu^{\prime 2}\right)}{k^{2}\left(k^{2}-h^{2}\right)}}=\dot{9}, 95861 .
\end{aligned}
$$

Mit diesen Constanten können nun zu jedem Werthe von $u$ die Coordinaten $x, y, z$ mittelst der Gleichungen (9) und (17) berechnet werden. Die folgende Tafel ist jedoch so angelegt, dass der eine Winkel $v$ als unabhängige Veränderliche genommen und mittelst der aus (10) und der Bedingung $u^{\prime}=0$ folgenden . Relation $\beta_{1,1} w=\beta_{2,1} v=0$ der

*) Die Logarithmen sind sïmmtlich Briggische. 
zugehörige Werth von $w$ berechnet wurde. Der Vollständigkeit halber sind die entsprechenden Werthe von $u$ ebenfalls notirt.

Die mit * bezeichneten Punkte sind die Berührungspunkte der geodätischen Linie mit der Krümmungslinie $\mu^{\prime}$. Da für sie $x_{1}=1$ sein muss, so erhält man die ihnen entsprechenden Werthe von $u$, indem man beachtet, dass in Gleichung $(8)$ b) $v\left(\begin{array}{ll}1 & 0 \\ 0 & 1\end{array}\right)(v \mid w)$ verschwindet.

\begin{tabular}{|c|c|c|c|c|c|}
\hline$u$ & $v \pi$ & $w \pi$ & $x$ & $y$ & $z$ \\
\hline 0 & 0 & 0 & $1,3160.7$ & 0 & 0 \\
\hline $0,2591.1$ & $20^{\circ}$ & $17^{0}, 015$ & $1,2641.8$ & $0,2135.7$ & $0,2121.7$ \\
\hline $0,5182.1$ & 40 & 34,030 & $1,1052.5$ & $0,4221.7$ & $0,4107.0$ \\
\hline $0,7773.2$ & 60 & 51,044 & $0,8352.2$ & $0,6136.1$ & $0,5752.1$ \\
\hline $1,1094.7^{*}$ & 85,636 & 72,855 & $0,3469.3$ & $0,7990.4$ & $0,6921.2$ \\
\hline $1,2955.3$ & 100 & 85,074 & $0,0336.5$ & $0,8667.4$ & $0,6947.0$ \\
\hline $1,5546.4$ & 120 & 102,09 & $-0,3951.5$ & $0,8623.0$ & $0,6197.1$ \\
\hline $1,8137.5$ & 140 & 119,11 & $-0,7623.4$ & $0,7909.5$ & $0,4712.8$ \\
\hline $2,0728.9$ & 160 & 136,12 & $-1,0342.9$ & $0,6562.6$ & $0,2790.6$ \\
\hline $2,3319.5$ & 180 & 153,13 & $-1,2031.7$ & $0,4747.5$ & $0,0694.8$ \\
\hline $2,5910.6$ & 200 & 170,15 & $-1,2717.3$ & $0,2572.4$ & $-0,1393.6$ \\
\hline $2,8501.9$ & 220 & 187,16 & $-1,2406.9$ & $0,0101.9$ & $-0,3335.2$ \\
\hline $3,1092.9$ & 240 & 204,18 & $-1,1059.2$ & $-0,2581.1$ & $-0,4967.4$ \\
\hline $3,3847.6^{*}$ & 261,27 & 222,27 & $-0,8477.4$ & $-0,5458.3$ & $-0,6119.5$ \\
\hline $3,6275.0$ & 280 & 238,21 & $-0,5363.0$ & $-0,7712.8$ & $-0,6424.7$ \\
\hline $3,8866.4$ & 300 & $25 \overline{7}, 22$ & $-0,1536.0$ & $-0,9474.6$ & $-0,5930.4$ \\
\hline $4,1457.3$ & 320 & 272,24 & $0,2307.7$ & $-0,0308.8$ & $-0,4667.4$ \\
\hline $4,4048.0$ & 340 & 289,25 & $0,5747.2$ & $-1,0144.2$ & $-0,2887.9$ \\
\hline $4,6638.9$ & 360 & 306,26 & $0,8439.6$ & $-0,9065.2$ & $-0,0877.6$ \\
\hline $4,9230.0$ & 380 & 323,28 & $1,0361.4$ & $-0,7189.5$ & $0,1139.4$ \\
\hline $5,1822.9$ & 400 & 340,30 & $1,1446.6$ & $-0,4691.4$ & $0,2964.6$ \\
\hline $5,4412.5$ & 420 & 357,32 & $1,1652.4$ & $-0,1654.4$ & $0,4435.2$ \\
\hline $5,7551.4^{*}$ & 444,23 & 377,93 & $1,0666.7$ & $0,2434.0$ & $0,5488.2$ \\
\hline $5,9595.0$ & 460 & 391,35 & $0,9310.0$ & $0,5089.1$ & $0,5625.6$ \\
\hline $6,2185.7$ & 480 & 408,36 & $0,6897.2$ & $0,8091.3$ & $0,5123.1$ \\
\hline $6,4777.1$ & 500 & 425,37 & $0,3940.4$ & $1,0337.1$ & $0,3934.3$ \\
\hline
\end{tabular}




\section{$\S 5$.}

\section{Specielle Fälle.}

Für den speciellen Fall $\mu^{\prime}=k$ fällt die geodätische Linie mit dem Hauptschnitt in der $x y$-Ebene zusammen. Denn je mehr sich $\mu^{\prime}$ dem Werthe $k$ nähert, desto mehr rücken die beiden Aeste der Krümmungslinie von zwei Seiten an den Hauptschnitt heran, und da die geodätische Linie das Gebiet zwischen diesen Aesten nicht überschreitet, so muss sie in der Grenzlage mit dem Hauptschnitte selbst zusammenfallen; die Gleichung (2) geht dann in die Summe aus einem elliptischen Integral 3. Gattung und einer Constanten über. Aehnliches gilt für den Hauptschnitt der $y z$-Ebene.

Für $\mu^{t}=h$ hingegen erhält mau, wie bereits erwähnt, sämmtliche durch die Kreispunkte gehende Linien, während Gleichung (2) in die Summe zweier elliptischer Integrale 3. Gattung übergeht. In der bereits in der Einleitung erwähnten Arbeit von Rohn*) wird nun diese Gleichung weiter behandelt, und schliesslich finden sich die rechtwinkligen Coordinaten eines Punktes der Kreispunktslinie durch elliptische Functionen zweier Parameter ausgedrückt, die mit einander durch eine Gleichung verbunden sind. Nun lassen sich aber diese Coordinaten, wie im allgemeinen Falle, direct durch Functionen eines einzigen Parameters darstelleu, wenn man sich ähnlicher Formeln bedient, wie sie Rosenhain im Eingang seiner Schrift bezüglich der Transscendenten 3. Gattung entwickelte**). Diese Formeln will ich im Folgenden angeben, da sie in engem Zusammenhang mit unserm Formeln einer allgemeinen geodätischen Linie stehen und auch für die Rechnung von Vortheil sein dürften.

Für $\mu^{\prime}=h$ wird Gleichung (2)

$$
\int_{\mu_{0}^{2}}^{\mu^{2}} \frac{\left.\sigma^{2}-\mu^{2}\right) d\left(\mu^{2}\right)}{\mu^{2}-h^{2} M_{h}}-\int_{0}^{\nu^{2}} \frac{\left(\sigma^{2}-\nu^{2}\right) d\left(\nu^{2}\right)}{\left(h^{2}-\nu^{2}\right) N_{h}}=0 .
$$

Hiebei bedeutet $\mu=\mu_{0}$ den Parameter des Schnittpunktes der Linie mit der $y z$-Ebene, während $M_{h}^{2}=\left(\sigma^{2}-\mu^{2}\right)\left(k^{2}-\mu^{2}\right) \mu^{2}$ und $N_{h}^{2}=\left(\sigma^{2}-\nu^{2}\right)$ $\cdot\left(k^{2}-\nu^{2}\right) \nu^{2}$ ist. Setzt man in dieser Gleichung $\mu^{2}=k^{2} x_{1}, \nu^{2}=k^{2} x_{2}$, so geht sie über in:

*) Die erste Arbeit, in welcher Formeln zur Berechnung einer solchen Linie aufgestellt werden, mag wohl eine aus dem Nachlasse von Jacobi durch Luther in Schumachers astronomischen Nachrichten Bd. 41, pag. 210 publicirte Abhandlung sein.

**) Rosenhain a. a. O. pag. 376 bis 380 . 


$$
\begin{gathered}
\int_{x_{0}}^{x_{1}} \frac{\left(1-x^{2} x_{1}\right) d x_{1}}{\left(1-\lambda^{2} x_{1}\right) V \overline{X_{1}}}+\int_{0}^{x_{2}} \frac{\left(1-x^{2} x_{2}\right) d x_{2}}{\left(1-\lambda^{2}, x_{2}\right) V X_{2}}=0, \\
X=x(1-x)\left(1-x^{2} x\right) ; \quad x^{2}=\frac{k^{2}}{\sigma^{2}} ; \quad \lambda^{2}=\frac{k^{2}}{h^{2}} ; \quad \mu_{0}=x^{2} x_{n} .
\end{gathered}
$$

Verbindet man hiermit die Gleichung

$$
\int_{0}^{x_{1}} \frac{d x_{1}}{V X_{1}}+\int_{0}^{x_{2}} \frac{d x_{2}}{V \overline{X_{2}}}=u_{1}+u_{2}=u,
$$

setzt $x_{0}=\sin$ am $u^{\prime}, \lambda^{2}=\sin ^{2}$ am $\varepsilon$ und bedient sich im Folgenden der bekannten Indicesbezeichnung der vier elliptisehen $\vartheta$-Functionen*), so erhält man leicht die Gleichung:

$$
\left.\left(u-u^{\prime}\right) \frac{\vartheta_{8}^{\prime}(\varepsilon)}{\vartheta_{3}(\varepsilon)}+\frac{1}{2} \log \frac{\vartheta_{1}\left(u_{1}-\varepsilon\right) \vartheta_{1}\left(u_{2}-\varepsilon\right) \vartheta_{1}\left(u^{\prime}+\varepsilon\right)}{\vartheta_{1}\left(u_{1}+\varepsilon\right) \vartheta_{1}\left(u_{2}+\varepsilon\right) \vartheta_{1}\left(u^{\prime}-\varepsilon\right)}+\frac{i \pi}{2}=0^{* *}\right) .
$$

Hieraus folgt, wenn man zur Abkürzung $\left(u-u^{\prime}\right) \frac{\vartheta_{3}^{\prime}(\varepsilon)}{\vartheta_{3}(\varepsilon)}=v$ und $\frac{\vartheta_{1}\left(u^{\prime}+\varepsilon\right)}{\vartheta_{1}\left(u^{\prime}-\varepsilon\right)}=\rho$ setzt,

$$
e^{2 v}=-\rho \frac{\vartheta_{1}\left(u_{1}-\varepsilon\right) \vartheta_{1}\left(u_{2}-\varepsilon\right)}{\vartheta_{1}\left(u_{1}+\varepsilon\right) \vartheta_{1}\left(u_{2}+\varepsilon\right)}
$$

Wendet man nun auf diese Gleichung die Additionsformeln an, oder transformirt man kürzer die von Rosenhain pag. 379 unter $\mathrm{Nr} .27$ und 380 unter Nr. 28 gegebenen Formeln, indem man daselbst $a=\varepsilon+i K^{\prime}$ und statt $e^{-v}$ überall $-\varrho e^{-v}$ setzt, so folgen die Formeln:

$$
\begin{gathered}
x \sqrt{x_{1} x_{2}}=\frac{\vartheta_{1}(\varepsilon)}{\vartheta(\varepsilon)} \frac{\rho e^{-v} \vartheta_{1}(u-\varepsilon)-e^{v} \vartheta_{1}(u+\varepsilon)}{\rho e^{-v} \vartheta(u-\varepsilon)+e^{v} \vartheta(u+\varepsilon)}, \\
\frac{x}{x_{1}} \sqrt{\left(1-x_{1}\right)\left(1-x_{2}\right)}=\frac{\vartheta_{2}(\varepsilon)}{\vartheta(\varepsilon)} \frac{\rho e^{-v} \vartheta_{3}(u-\varepsilon)-e^{v} \vartheta_{3}(u+\varepsilon)}{\rho e^{-v} \vartheta(u-\varepsilon)+e^{v} \vartheta(u+\varepsilon)}, \\
\frac{1}{x_{1}} \sqrt{\left(1-x^{2} x_{1}\right)\left(1-x^{2} x_{2}\right)}=\frac{\vartheta_{3}(\varepsilon)}{\vartheta(\varepsilon)} \cdot \frac{\rho e^{-v} \vartheta_{2}(u-\varepsilon)-e^{v} \vartheta_{2}(u+\varepsilon)}{\rho e^{-v} \vartheta(u-\varepsilon)+e^{v} \vartheta(u+\varepsilon)} \\
\sqrt{-\left(1-\lambda^{2} x_{1}\right)\left(1-\lambda^{2} x_{2}\right)}=\frac{\vartheta(0) \vartheta_{2}(\varepsilon) \vartheta_{3}(\varepsilon) V \varrho}{\vartheta_{2}(0) \vartheta_{3}(0) \vartheta_{1}(\varepsilon)} \cdot \frac{2 \vartheta_{1}(u)}{\rho e^{-v} \vartheta(u-\varepsilon)+e^{v} \vartheta(u+\varepsilon)}
\end{gathered}
$$

Wendet man dann auf die Gleichungen (4) $\mu^{2}=\nu^{2}=k^{2} x$ an und führt aus obigen Formeln die hiedurch auftretenden symmetrischen Functionen von $x_{1}, x_{2}$ ein, so ergiebt sich für die rechtwinkligen Coordinaten eines Punktes der geodätischen Linie:

*) Vergl. Jacobi, Werke, neue Ausgabe, Bd. I, pag. 501. Oder Rosenhain a. a. O. pag. 368 .

**) Diese Gleichung stimmt bis aut die Bezeichnung mit der von Rohn a. a. 0 . entwickelten überein. 


$$
\begin{aligned}
& x=\begin{array}{lll}
\sigma^{2} & \vartheta_{1}(\varepsilon) & \varrho e^{-v} \vartheta_{1}(u-\varepsilon)-e^{v} \vartheta_{1}(\mu+\varepsilon) \\
k^{2} & \vartheta(\varepsilon) & \varrho e^{-v} \vartheta(\mu-\varepsilon)+e^{v} \vartheta(\mu+\varepsilon)
\end{array}, \\
& y=h^{2} \sqrt{\frac{\sigma^{2}-h^{2}}{k^{2}-h^{2}}} \frac{\vartheta_{2}(\varepsilon) \vartheta_{3}(\varepsilon) \vartheta(0)}{\vartheta_{2}(0) \vartheta_{3}(0) \vartheta_{1}(\varepsilon)} \sqrt{\rho} \frac{2 \vartheta_{1}(u)}{\rho e^{-v} \vartheta(u-\varepsilon)+e^{v} \vartheta(u+\varepsilon)}, \\
& z=\sqrt{k^{2}-h^{2} \vartheta_{2}(\varepsilon)} \frac{\rho e^{-v} \vartheta_{3}(u-\varepsilon)-e^{v} \vartheta_{1}(u+\varepsilon)}{\mathfrak{v}(\varepsilon)} \frac{\rho e^{-v} \vartheta(u-\varepsilon)+e^{v} \vartheta(u+\varepsilon)}{} .
\end{aligned}
$$

Hierdurch sind, wie im allgemeinen Falle, die rechtwinkligen Coordinaten durch die eine Variable $u$ ausgedrückt.

Eine Vereinfachung erfahren diese Formeln noch, wenn man die Linie durch den Endpunkt der mittleren Axe gehen lässt, wodurch dieselbe nicht specialisirt wird. Dann ist nämlich $u^{\prime}=K=\int_{0}^{1} \frac{d x}{V \bar{X}}$, somit $v=(K-u) \frac{\vartheta_{3}^{\prime}(\varepsilon)}{\vartheta_{3}(\varepsilon)}$ und $\varrho=1$. Für die in der Formel von $y$ auftretende Constante ergiebt sich dann leicht ein vereinfachter Werth, so dass man hat:

$$
\begin{aligned}
& x=\frac{\sigma^{2}}{k} \frac{\vartheta_{1}(\varepsilon)}{\vartheta(\varepsilon)} \frac{e^{-v} \vartheta_{1}(u-\varepsilon)-e^{v} \vartheta_{1}(u+\varepsilon)}{e^{-v} \vartheta(u-\varepsilon)+e^{v} \vartheta(u+\varepsilon)}, \\
& y=h \sqrt{\sigma^{2}-h^{2}} \cdot \sqrt{\frac{\sigma \pi}{k h}} \vartheta_{3}(\varepsilon) \frac{2 \vartheta_{1}(u)}{e^{-v} \vartheta(u-\varepsilon)+e^{v} \vartheta(u+\varepsilon)},
\end{aligned}
$$

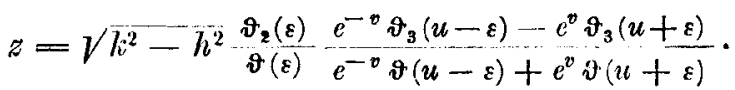

$\S 6$.

Gleichungen für eine Enveloppe geodätischer Linien und Discussion derselben.

An die bereits in der Einleitung erwähnte Bemerkung von J a cobi, dass ein Büschel geodätischer Linien auf positiv gekrümmten Flächen im Allgemeinen eine Enveloppe besitzt, anknüpfend, hat O. Bonnet*) gezeigt, dass dies bei allen geschlossenen positiv gekrümmten Flächen eintreten muss. Auf solchen Flächen sind also dann alle geodätischen Linien nur bis zu einer gewissen Grenze, nämlich bis zum Berührungspunkte mit ihrer gemeinsamen Enveloppe kürzeste Linien. Für das dreiaxige Ellipsoid lässt sich eine solche Enveloppe auf fơlgende Art durch Gleichungen bestimmen:

Setzt man den Werth der Constanten $u^{\prime}$ in Gleichung (2) $\$ 1$.

*) Sur quelques propriétés des lignes géodésiques. Comptes rendus de l'Académie des Sciences de Paris. t. 40, pag. 1311-1313. 
dadurch fest, dass man die geodätische Linie durch den Punkt $\mu=\mu_{0}$, $\nu=\nu_{0}$ gehen lässt, so nimmt die Gleichung die Gestalt an :

$$
\int_{\mu_{0}^{2}}^{\mu^{2}} \frac{d\left(\mu^{2}\right)\left(\sigma^{2}-\mu^{2}\right)}{M} \pm \int_{\nu_{0}^{2}}^{\nu^{2}} \frac{d\left(\nu^{2}\right)\left(\sigma^{2}-v^{2}\right)}{N}=0 .
$$

Hier hat man sich des unteren Zeichens zu bedienen, wenn $\mu$ und $v$ zugleich wachsen oder abnehmen, des oberen im Gegenfalle. Da durch den Parameter $\mu^{2}$, der in $M$ und $N$ auftritt, die einzelnen Linien des Büschels mit dem Mittelpunkte $\mu_{0}, \nu_{0}$ individualisirt sind, so biitte man obige Gleichung nach $\mu^{\prime 2}$ zu differentiiren und aus dem Differential und der vorliegenden Gleichung $\mu^{\prime 2}$ zu eliminiren, um die Gleichung der Enveloppe in elliptischen Coordinaten zu erhalten. Die durch Differentiation resultirende Gleichung ist:

$$
\int_{\mu_{0}^{2}}^{\mu^{2}} \frac{\left(\sigma^{2}-\mu^{2}\right) d\left(\mu^{2}\right)}{\left(\mu^{2}-\mu^{2}\right) \bar{M}} \mp \int_{\nu_{0}^{2}}^{v^{2}} \frac{\left(\sigma^{2}-\nu^{2}\right) d\left(\nu^{2}\right)}{\left(\mu^{2}-\nu^{2}\right) N}=0,
$$

und da eine Elimination natürlich explicite nicht möglich ist, so müssen uns die beiden Gleichungen (19) und (20) miteinander die Curve bestimmen.

Aus dem Büschel geodätischer Linien greifen wir jetzt eine heraus, für welche $\mu^{\prime}$ zwischen den Grenzen $v=0$ und $\nu=h$ liegen möge, dann ist $\nu_{0}>\mu$, und lässt man die Linie von einem Punkte beschreiben, der seine Bewegung in $A$ beginnt, so wird, welche Werthe auch $\mu$ durchläuft, das erste Integral in (20) sein Zeichen beibehalten, da es für die angenommenen Grenzen von $\mu^{\prime}$ weder Null noch unendlich werden kann. Setzt man also den Anfangswerth beider Integrale als positiv voraus und lässt den die Linie beschreibenden Punkt von $\boldsymbol{A}$ gegen dén Hauptschnitt der mittleren und kleinen Axe emporsteigen, während er sich zugleich dem Hauptschnitte der grossen und kleinen Axe nähert, (in der Pfeilrichtung von $A$ nach $B$ in Fig. 2), so nimmt anfangs $\mu$ gegen $h$ und $\nu$ gegen $0 \mathrm{ab}$, die beiden Integrale haben also gleiches, positives Zeichen, und folglich ist in (19) das obere Zeichen zu nehmen. Während nun das erste Integral stets positiv bleibt, wächst das zweite von Null für $v=\nu_{0}$ beginnend bis $+\infty^{*}$ ) (fürr $\nu=\mu^{\prime}$, Punkt $B$ in Fig 2.), springt in diesem Punkte auf $-\infty$ über und langt in $\nu=\nu_{0}^{\prime}$ (Punkt $C$ ) mit dem positiven

*) Das Integral wird in Folge des Factors $\frac{1}{\left(\mu^{\prime 2}-\nu^{2}\right)^{\frac{3}{2}}}$ für $\nu=\mu^{\prime}$ unstetig und zwar von der Ordnung $\frac{3}{2}$, somit wechselt es in diesem Punkte sein Zeichen und springt von $+\infty$ auf $-\infty$ über. 
Periodicitätsmodul $\left.J_{v}=2 \int_{\nu_{0}^{2}}^{\mu^{\prime 2}} \frac{d\left(\nu^{2}\right)\left(\sigma^{2}-\nu^{2}\right)}{\left(\mu^{2}-\mu^{2}\right)} *\right)$ an. Hierbei bedeutet $\nu_{0}{ }^{\prime}=\nu_{0}$ den andern Zweig der Krümmungslinie $\nu_{0}$, auf welchem der Ausgangspunkt $A$ nicht liegt.

' $\mathrm{W}$ wischen $\nu=\mu^{\prime}$ und $\nu=\nu_{0}^{\prime}$ hat also das zweite Integral negative Werthe; die Gleichung (20) kann also nur für solche Werthe von $\nu$ befriedigt werden, welche nach der Culmination der Linie in $\nu=\mu^{\prime}$ und vor Erreichung der Krümmungslinie $v_{0}^{\prime}$ liegen, also liegt der Berührungspunkt $X$ der betreffenden geodätischen Linie mit ihrer Fnveloppe zwischeu diesen Grenzen.

Unter allen Linien, die von $A$ ausgehen, befindet sich eine, die dje Krümmungslinie $\nu_{0}$ in $A$ berührt, für sie ist also $\mu^{\prime}=\nu_{0}$; ich behaupte, ihr Berührungspunkt mit der Enveloppe tritt daun ein, wenn die Linie den zweiten Ast $\nu_{0}^{\prime}$ der Krümmungslinie tangirt. Denn nimmt man $\mu^{\prime}$ wenig verschieden $* *$ ) von $\nu_{0}$, so liegt, wie eben gezeigt, der Berührungspunkt dieser Linie mit der Enveloppe nach $\nu=\mu^{\prime}$ und vor $\nu=\nu_{0}^{\prime}$. Näliert sie sich also der in $A$ tangirenden Geodätischen, so rückt $\mu^{\prime} \operatorname{dem} \nu_{0}^{\prime}=\nu_{0}$ unendlich wahe, und folglich nähert sich der Berührungspunkt $X$ zugleich mit dem Punkte $B$ immer mehr dem Punkte $C$ auf der Krümmungslinie $\nu_{0}^{\prime}$, um in der Grenze mit ihm auf $\nu_{0}^{\prime}$ zusammenzufallen. Das Gleiche gilt natürlich auch für jene geodätische Linie des Büschels, welche in $A$ die Krümmungslinie $\mu=\mu_{0}$ berührt; ihr Berührungspunkt mit der Enveloppe tritt dann ein, wenn die geodätische Curve den zweiten Ast $\mu_{0}^{\prime}$ tangirt.

Nun scheiden aber diese beiden Geodütischen das Büschel in $A$ gleichsam in 4 Quadranten, und da jede mit der Krümmungslinie $\mu_{0}^{\prime}$ oder $\boldsymbol{\nu}_{0}^{\prime}$, wie uımittelbar einzusehen, zwei Berührungspunkte haben wird, da sie nach zwei Richtungen von $A$ ausläuft, so sind hierdurch 4 Enveloppenpunkte bestimmt, die analog den vier Quadranten die Einhüllende in vier Theile scheiden werden. In der That: in jedem solchen Punkte schliessen sich zwei Zweige der Enveloppe tangential an die Krümmungslinie $\mu_{0}^{\prime}$ resp. $\nu_{0}^{\prime}$ an, da man von $A$ aus zu der im Ausgangspunkte berührenden Curve zwei ${ }^{* * *}$ ) in verschiedene Quadranten auslaufende, ihr unendlich benachbarte Linien ziehen

*) Diesen Modul kann man durch partielle Integration so umgestalten, dass er für $\nu=\mu^{\prime}$ nicht mehr $\infty$ wird; man erhält dann:

$$
J_{0}=\frac{2}{\mu^{\prime 2}} \int_{\mu^{\prime 2}}^{\nu^{2}} \frac{\nu}{\sqrt{\mu^{\prime 2}-\nu^{2}}} \cdot \frac{\left(\sigma^{2}-\nu^{2}\right)\left(h^{2}-\nu^{2}\right)+\left(\sigma^{2}-h^{2}\right)\left(k^{2}-\nu^{2}\right)}{\sqrt{\left(\sigma^{2}-\nu^{2}\right)\left(k^{2}-\nu^{2}\right)^{3}\left(h^{2}-\nu^{2}\right)^{3}}} d\left(\nu^{2}\right),
$$

und da $\mu^{\prime}<v_{0}$ ist, so muss $J_{0}$ positiv sein.

**) Vergl. v. Mangoldt, a. a. O. pag. 48, Anmerk.

***) Vergl. § 1. am Ende. 
kann, welche sich von zwei verschiedenen Seiten her an sie anschliessen (vergl. Fig. 2). Diese vier Punkte sind massgebend sowohl für die Gestalt als auch für die Lage der Enveloppe. Wir haben also zunächst ihre gegenseitige Stellung in's Ange zu fassen.

\section{$\S 7$.}

Aufstellung eines allgemeinen Satzes über die Lage des Berührungspunktes einer geodätischen Linie mit ihrer Enveloppe und Gestalt der letzteren.

Wir beweisen vorerst folgende Ungleichungen :

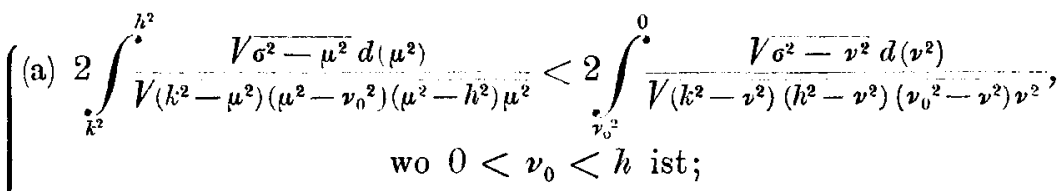

$$
\begin{aligned}
& \text { (b) } 2 \int_{\mu_{0}^{2}}^{k^{2}} \frac{V \sqrt{\sigma^{2}-\mu^{2}} d\left(\mu^{2}\right)}{V\left(k^{2}-\mu^{2}\right)\left(\mu^{2}-\mu_{0}^{2}\right)\left(\mu^{2}-h^{2}\right) \mu^{2}}<2 \int_{0}^{h^{2}} \frac{d\left(\nu^{2}\right) V \sigma^{2}-\nu^{2}}{V\left(k^{2}-\nu^{2}\right)\left(h^{2}-\nu^{2}\right)\left(\mu_{0}^{2}-\nu^{2}\right) \nu},
\end{aligned}
$$

Dabei sind die Integrale so zu verstehen, dass die Integrationsvariable von der untern Grenze beginnend, die obere umläuft und wieder nach ihrem Ausgangspunkte zurückkehrt.

Um die erste Ungleichung zu beweisen, vergrössern wir das Integral links, indem wir statt $\sqrt{\sigma^{2}-\mu^{2}} \sqrt{\sigma^{2}-h^{2}}$ setzen, während wir zur Verkleinerung des zweiten statt $\sqrt{\sigma^{2}-v^{2}} \sqrt{\sigma^{2}-\nu_{0}^{2}}$ einführen. Hierdurch gehen die beiden Integrale in elliptische der ersten Gattung über. Un diese bezüglich ihrer Grösse zu vergleichen, setzen wir links $\mu^{2}=\frac{h^{2}\left(k^{2}-v_{0}^{2}\right)-\nu_{0}^{2}\left(k^{2}-h^{2}\right) y}{h^{2}-\nu_{0}^{2}-\left(k^{2}-h^{2}\right) y}$ und rechts $v^{2}=\frac{k^{2} y_{0}^{2}}{k^{2}-\nu_{0}^{2}-\nu_{0}^{2} y} ;$ dann gehen sie beide in vollständige Integrale mit dem nämlicheu Modul $x^{2}=\frac{\nu_{0}^{2}\left(k^{2}-h^{2}\right)}{h^{2}\left(k^{2}-\nu_{0}^{2}\right)}$ über und unterscheiden sich nur durch die coustanten Factoren, so dass man mit Benutzung der Jacobi'schen Bezeichnung für das vollständige Integral erster Gattung hat:

$$
\frac{2}{h} \sqrt{\frac{\sigma^{2}-h^{2}}{k^{2}-\nu_{0}^{2}}} K<\frac{2}{\nu_{0}} \sqrt{\frac{\overline{\sigma^{2}}-\nu_{0}^{2}}{k^{2}-\nu_{0}^{2}}} K,
$$

was unmittelbar einleuchtet, da $\nu_{0}<h$ voransgesetzt wurde. Also muss um so mehr obig'e Ungleichung (a) bestehen.

Zum Beweise der zweiten Ungleichung sei das Integral links vergrössert, indem man statt $\sqrt{\sigma^{2}-\mu^{2}} \sqrt{\sigma^{2}-\mu_{0}^{2}}$ einführt, und das Integral rechts verkleinert, indem man an Stelle von $\sqrt{\sigma^{2}-v^{2}}$ $\sqrt{\sigma^{2}-h^{2}}$ setzt. Macht man dann resp. die Substitutionen 


$$
\mu_{2}=\frac{k^{2} \mu_{0}^{2}}{\mu_{0}^{2}+\left(k^{2}-\mu_{0}^{2}\right) y} \text { und } \quad \nu^{2}=-\frac{k^{2} h^{2} y}{k^{2}-h^{2}+h^{2} y}
$$

so erhält man, wie oben, zwei elliptische Integrale mit dem gleichen Modul $x^{2}=\frac{h^{2}\left(k^{2}-\mu_{0}^{2}\right)}{\mu_{0}^{2}\left(k^{2}-h^{2}\right)}$, und es besteht die Ungleichung:

$$
\frac{2}{\mu_{0}} \sqrt{\frac{\sigma^{2}}{h^{2}-\mu_{0}^{2}}} K<\frac{2}{\mu_{0}^{2}} \sqrt{\frac{\sigma^{2}-h^{2}}{k^{2}-h^{2}}} \cdot K,
$$

da $\mu_{0}>h$ ist. Somit ist auch die Richtigkeit der zweiten Ungleichheit um so mehr einleuchtend.

Verfolgt man jetzt die geodätische Linie, welche $\nu_{0}$ im Ausgangspunkte $A$ berührt, indem man den $Z$ weig betrachtet, $\operatorname{der}$ von $A$ aufwärts ïber den Hauptschnitt der grossen und kleinen Axe emporsteigt, so wird die eine Coordinate $\nu$ des die Linie beschreibenden Punktes von $v=\nu_{0}$ äber $\nu=0$ nach $\nu=\nu_{0}^{\prime}$ laufen (vergl. Fig. 2), um $\nu_{0}^{\prime}$ in $A^{\prime}$ zu tangiren, das zweite Integral in Gleichung (19) hat dann

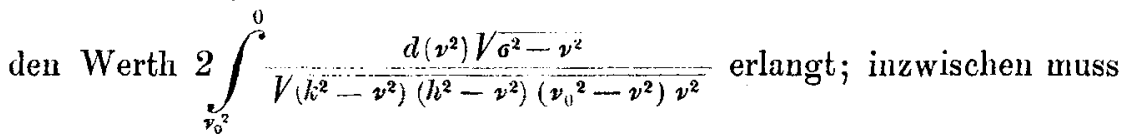
aber die andere Coordinate des Punktes von $\mu=\mu_{0}$ über $\mu=h$ und $\mu=k$ nach $\mu=\mu_{0}^{\prime}$ gelangt und diesen Werth bereits ïberschritten haben, da der bis zu $\mu=\mu_{0}^{\prime}$ erlangte Integralwerth

$$
2 \int_{k^{2}}^{h^{2}} \frac{\sqrt{\sigma^{2}-\mu^{2}} d\left(\mu^{2}\right)}{\sqrt{\left(k^{2}-\mu^{2}\right)\left(\mu^{2}-\nu_{0}^{2}\right)\left(\mu^{2}-h^{2}\right) \overline{\mu^{2}}}}
$$

nach der ersten Ungleichung in (20) noch kleiner ist als

$$
2 \int_{\nu_{0}^{2}}^{v^{2}} \frac{d\left(\nu^{2}\right) \sqrt{\sigma^{2}-\nu^{2}}}{V\left(k^{2}-\nu^{2}\right)\left(\nu_{0}^{2}-\nu^{2}\right)\left(h^{2}-\nu^{2}\right) \nu^{2}},
$$

den er doch erreiehen soll. Also kann der Berührungspunkt der geodätischen Linie mit $\nu_{0}^{\prime}, d . h$. der eine dieser vier ausgezeichneten Enveloppenpunkte, erst dann auftreten, wenn die Linie $\mu_{0}^{\prime}$ (von oben kommend) bereits durchsetzt hat*).

Genau ebenso zeigt sich, wenn man jenen Zweig der Linie in's Auge fasst, der von $A$ abwärts dem Hauptschnitte der grossen und mittleren Axe zustrebt, dass derselbe von unten kommend $\mu_{0}^{\prime}$ bereits durchsetzt hat, bevor er $\nu_{0}^{\prime}$ berührt. Die beiden Berïlırungspunkte der in

*) Dieser Satz stimmt mit einem erst vor Kurzem in einer Abhaudlung: "Ueber Fidenconstructionen des Ellipsoides", diese Annalen Bd. XX, pag. 160, von Herrn Staude yeröffentlichten überein. Er war mir schon lange Zeit bekannt, und da mein Beweis sich wesentlich von dem dort pag. 161 gegebenen unterscheidet, so erlaubte ich $\mathrm{mir}$, ihn mitzutheilen. 
$A$ die Krümmungslinie $v_{0}$ tangirenden geodätischen Linic mit $v_{0}^{\prime}$ oder dic Berïhrungspunkte der Enveloppe mit dieser Krïmmungslinic liegen somit zu verschiedenen Seiten der Krïmmungslinie $\mu_{0}^{\prime}$.

Fasst man ferner jene Curve in's Auge, welche $\mu_{0}$ im Ausgangspunkte berïhrt, und zwar den Zweig derselben, welcher sich von $A$. gegen den Hauptschnitt der mittleren und kleinen Axe erstreckt, so muss $\mu$, bis es die Linie $\mu_{0}^{\prime}$ tangirt, von $\mu_{0}$ über $k$ nach $\mu_{0}^{\prime}$ gegangen sein; somit hat das erste Integral in (19) den Werth

$$
2 \int_{\mu_{0}{ }^{2}}^{k^{2}} \frac{d\left(\mu^{2}\right) V \overline{\sigma^{2}-\mu^{2}}}{V\left(k^{2}-\mu^{2}\right)\left(\mu^{2}-\mu_{0}{ }^{2}\right)\left(\mu^{2}-h^{2}\right) \mu^{2}}
$$

erlangt. Damit nun aber auch das zweite lutegral den gleichen Werth bekommt, darf $\nu$ von $\nu_{0}$ über 0 und $h$ laufend den Werth $\nu_{0}{ }^{\prime}$ noch nicht erlangt haben, da sonst das Integral auf

$$
2 \int_{0}^{h^{2}} \frac{d\left(\nu^{2}\right) \sqrt{\sigma^{2}-\nu^{2}}}{\sqrt{\left(k^{2}-\nu^{2}\right)\left(h^{2}-\nu^{2}\right)\left(\mu_{0}^{2}-\nu^{2}\right) \nu^{2}}}
$$

angewachsen wäre, welcher Werth nach der zweiten Ungleichung in (21) grösser als

$$
2 \int_{\mu_{0}^{2}}^{k^{2}} \frac{d\left(\mu^{2}\right) \sqrt{\sigma^{2}-\mu^{2}}}{\sqrt{\left(k^{2}-\mu^{2}\right)\left(\mu^{2}-\mu_{0}^{2}\right)\left(\mu^{2}-h^{2}\right) \mu^{2}}}
$$

ist. Also tritt der fragliche Berührungspuntet der Linie mit $\mu_{0}^{\prime}$ ein, bevor sie $\nu_{0}^{\prime}$ schneidet. Ganz ebenso folgt, dass der zweite Zweig der Linie, welcher von $A$ gegen den Hauptschnitt der grossen und kleinen Axe strebt, $\mu_{i}{ }^{\prime}$ berührt, bevor er $\nu_{0}^{\prime}$ erreicht. Also liegen auch die zwei Berïhrungspunkte der Enveloppe mit $\mu_{0}^{\prime} z u$ verschiedenen Seiten von $\nu_{0}^{\prime}$.

Da nun die geodätischen linien des Büschels continuirlich auf einander folgen, so sind diese vier charakteristischen Punkte durch vier Zweige mit einander verbunden und zwar $\mathrm{zu}$ einer ähnlichen sternförmigen Figur wie auf den Rotationsellipsoiden. Je ein solcher Zweig kommt durch alle diejenigen Linien zu Stande, welche von $A$ aus in einen der oben erwähnten vier Quadranten eintreten. Dabei ist nach diesen Betrachtungen klar, dass sämmtliche geodätische Linien, die eine Krümmungslinie des Systemes $v$ tangiren, ihre EnveJoppe erst dann berühren, wenn sie den Werth $\mu_{0}^{\prime}$ überschritten haben, dass hingegen zwei unendlich benachbarte Linien des ersten Systems, welche eine Krümmungslinie $\mu$ berühren, schon zusammentreffen, bevor sie noch $\nu_{0}^{\prime}$ erreicht haben. Die Linie durch die Kreis- 
punkte, welche zu beiden Systemen gehört, geniesst natürlich auch beide Eigenschaften.

Geht eine geodätische Linie von einem Punkte der Krümmungslinie $\mu_{0}$ oder $\nu_{0}$ aus, berührt dann $\mu^{\prime}$, das zum einen oder andern System gehören kann, durchschneidet $\mu_{0}$ resp. $\nu_{0}$ abermals und erstreckt sich bis zum zweiten Aste der Krümmunglinie $\mu_{0}^{\prime}$ oder $v_{0}^{\prime}$, so nenne ich das so beschriebene Stück der Linie ilhro halbe Periode*) (Fig 2 das Stück $A B D$ ). Mit Einführung dieser Bezeichnung kann man dann die durch obige Untersuchung gewonnenen Resultate in folgendem Satze vereinigen: "Alle geodätischen Linien cines Ellipsoides, lic von eincm Punlite ausgehen, bilden eine Enveloppe und zwar treffen die Linien des ersten Systems ithre Enveloppe vor Vollendung ihrer halben Perioden, während dies für geodätische Linien des zwciten Systems erst nach Vollendung ihrer halben Perioden eintritt.“*:*)

\section{$\S 8$.}

Grenzen, bis zu welchen die drei Hauptschnitte des Ellipsoids kürzeste Linien sind.

Bezeichnet man der Kürze halber die drei Hauptschnitte mit ihren elliptischen Coordinaten (vergl. § 1.) und verlegt den Büschelmittelpunkt $A$ in irgend einen Puukt des Axenschnittes $\mu=k$, so ist die Lage dieses Punktes auf demselben durch einen gewissen Werth $\nu_{0}$ bestimmt. Die $v_{0}$ im Ausgangspunkte berührende geodätische Linie tangirt dann $\nu_{0}^{\prime}$ nach Vollendung ihrer halben Periode, die Enveloppe liegt symmetrisch in Bezug auf den Axenschnitt, zwei ihrer Spitzen befinden sich auf demselben, während die andern zwei auf $\nu_{0}^{\prime}$ liegen. Rückt $A$ in den Endpunkt der mittleren Axe, so fallen $\nu_{0}$ und $v_{0}$ ' mit $v=0$ zusammen, und die zwischen ihnen sich bewegende geodätische Linie geht in den Axenschnitt selbst über, derselbe ist daber geodätische Linie; da er aber seine Enveloppe nach Vollendung seiner halben Periode, das heisst nachdem er $\mu=k$ durchsetzt hat, berührt, so ist er in einer Ausdelınung kürzeste Linie, die grösser als die halbe Peripherie der von ihm ausgeschnittenen Ellipse ist. Da ferner für dieselbe Lage von $A \mu_{0}$ mit $\mu=k$ zusammenfällt, diese Linie aber vor Erreichung der halben Periode die Enveloppe berührt, so ist der Axenschnitt $\mu=k$ in einer Ausdehnung kürzeste |Linie, die kleiner als der halbe Ellipsenumfang ist.

*) Vergl. meinen Aufsatz a. a. O. pag. 558.

**) Dieser Satz entspricht genau dem in meinem Aufsatze pag. 561 für beide Rotationsellipsoide gegebenen. Das dreiaxige Ellipsoid besitzt eben zwei Systeme geodätischer Linien, wovon das eine die Eigenschaften der Linien auf dem verlängerten, das andere die der Linien anf dem abgeplatteten Ellipsoide geniesst. 
Wandert jetat $A$ auf $\nu=0$ weiter, so wird seine Lage durch eine gewisse Krümmungslinie $\mu_{0}$ festgelegt, und die Grenzpunkte, bis $\mathrm{zu}$ welchen die $\mu_{0}$ berührende Geodätische kürzeste linie ist, d. h. die auf ihr liegenden Enveloppenspitzen treten stets auf, bevor sie $\nu=0$ wieder trifft. Langt daher $A$ im Endpunkte der kleinen Axe an, so folgt, dass der Hauptschnitt $\mu=h$ von diesem Punkte aus gerechnet in einer kleineren Ausdehnung kürzeste Linie ist als der halbe Ellipsenbogen beträgt, während der Schnitt $v=0$ die nämliche Eigenschaft behielt, die er für den Endpunkt cler mittleren Axe als Ausgangspunkt der Linien besass.

Rückt der Büschelmittelpunlst auf $\mu=h$ gegen den Endpunkt der grossen Axe, so wird seine Lage bis zum Kreispunkte durch eine Coordinate $\nu=\nu_{0}$ bestimmt, und die Greuzpunkte der $\nu_{0}$ berthrenden Linie liegen also nach Vollendung der halben Perioden, d. h. wachdem die Linie bereits $\nu=h$ wieder durchschnitten hat, während die beiden andern Spitzen dem Hauptschnitte angehören und so liegen, dass derselbe stets in einer kleineren Ausdehnung kürzeste Linie ist, als die halbe Ellipsenperipherie; er rechnet ja von $\nu=0$ bis $\nu=h$ zum Systeme der Krümmungslinien $\mu$. Dabei zeigt sich ferner, dass die Enveloppe sich immer mehr zusammenzieht, bis $A$ den Kreispunkt erreicht, in welchem Falle sämmtliche Geodätischen des Büschels $A$ durch den entgegengesetzten Kreispunkt laufen, welcher dann die Enveloppe reprïsentirt.

Bewegt sich $A$ noch weiter gegen den Endpunkt der grossen Axe, so wird seine Lage durch eiven Werth $\mu=\mu_{0}$ festgelegt, und folglich liegen die Spitzen auf der $\mu_{0}$ tangirenden geodätischen Linie stets vor Vollendung der Periode, d. h. bevor sie $\mu=h$ zum zweiten Male trifft, während $\mu=h$ in einer die Länge des halben Ellipsenbogens übertreffenden Ausdehnung kürzeste Linie ist, da dieser Hauptschnitt vom Kreispunkte bis zum Endpunkte der grossen Axe der Schaar der Krümmungslinien $\nu$ angehört.

Fällt endlich der Büschelmittelpunkt mit dem Endpunkte der grossen Axe zusammen, so folgt, dass $\mu=z$ in einer Länge kürzeste Linie ist, die die halbe Ellipsenperipherie nicht erreicht.

Fassen wir diese Resultate in einen Satz, so folgt: "Die beiden Hauptschnitte der grossen und mittleren, der mittleren und kleinen Axe sind von irgend einem ihrer Punkte aus gerechnet in einer Ausdehnung kürzeste Linien, welche bezüglich die halbe Ellipsenperipherie (dieser Schnitte) nicht erreicht oder übertrifft. Der Axenschnitt der grossen und kleinen Axe hingegen ïbertrifft als kürzeste linie aufgefasst die halbe Ellipsenperipherie an Länge, wenn man von einem Punlte aus zählt, der dem durch zwei Kreispunkte begrenzten Stücke angehört, welches den Endpunlt der grossen Axe enthält, während er von seinen 
übrigen Punkten aus gerechnet in einer Längc, kleiner als der halbe Ellipscnumfang, lï̈rzeste Linie ist." *)

\section{$\S 9$.}

\section{Bestimmung der Enveloppe durch hyperelliptische Functionen.}

Will man Gleichungen, um die einzelnen Punkte einer Enveloppe rechnerisch zu bestimmen, oder will man mit andern Worten angeben, wie weit irgend eine vorgelegte geodätische Linie, von irgend einem Punkte aus gerechnet kürzeste Linie ist, so kann man sich diese leicht folgendermassen verschaffen. Fasst man nämlich die Gleichungen in's Auge:

$$
\left\{\begin{array}{l}
\text { a) } \int_{k^{2}}^{\mu^{2}} \frac{d\left(\mu^{2}\right)}{\bar{M}} \pm \int_{h^{2}}^{r^{2}} \frac{d\left(\nu^{2}\right)}{N}=u, \\
\text { b) } \int_{k^{2}}^{\mu^{\mu^{2}}} \frac{d\left(\mu^{2}\right)\left(\sigma^{2}-\mu^{2}\right)}{\left(\mu^{2}-\mu^{\prime 2}\right)-M^{-}}+\int_{h^{2}}^{v^{2}} \frac{d\left(v^{2}\right)\left(\sigma^{2}-v^{2}\right)}{\left(\mu^{\prime 2}-\nu^{2}\right) N}=u^{\prime \prime},
\end{array}\right.
$$

von denen die erste mit Gleichung (5) übereinstimmt, während die zweite mit Gleichung (19) zusammenfällt, indem die Constante $u$ " nur von der Wahl des Ausgangspunktes abhängt, so kann man mit Hilfe von a) die zweite Gleichung, die aus zwei hyperelliptischen Integralen zweiter Gattung besteht, in die Form bringen:

$$
\left.A v+A_{1} w+c_{1} \frac{\partial \log v\left(\begin{array}{ll}
1 & 0 \\
0 & 1
\end{array}\right)(v \mid w)}{\partial v}+c_{2} \frac{\partial \log v\left(\begin{array}{ll}
1 & 0 \\
0 & 1
\end{array}\right)(v \mid w)}{\partial w}=C ;^{* *}\right)
$$

$v$ und $w$ hängen durch die Gleichungen (10) von $u$ ab. Die Constanten lassen sich durch $\mathfrak{g}$-Quotienten mit verschwindenden Argumenten ausdrücken in Verbindung mit den Grössen $\beta_{p, q}$ des $\S 3$.***)

Giebt man nun einen bestimmten Werth von $\mu^{\prime}$, d. h. wäblt man eine geodätische Linie des Büschels aus, so sind damit die Constanten in Gleichung (23) bestimmt. Löst man daher diese Gleichung nach $u$ auf und führt den gefundeneu Werth $u_{0}$ in die Gleichungen (9) der geodätischen Linien ein, so hat man die rechtwinkligen Coordinaten des Berührungspunktes der Linie mit ihrer Enveloppe.

*) Diesem Satze entspricht ein ähnlicher für die beiden Rotationsellipsoide, lautend: „Der Aequatorkreis eines verlängerten Ellipsoides ist in einer Ausdehnung, kleiner als der Halbkreis, der Aequator eines Sphäroids aber in einer Länge, grösser als derselbe, kürzeste Linie.

**) Weierstrass a. a. O. pag. 995 giebt eine ähnliche Gleichung zur Bestimmung der Länge eines geodätischen Bogens.

***) Vergl. L. Schleiermacher: "Ueber die Bewegung eines schweren Punktes auf dem verlängerten Rotationsellipsoide". Inauguraldiesertation, Erlangen, pag. 18. 


\section{$\S 10$.}

Geodätische Linien auf dem zweischaligen Hyperboloide.

Auf dem zweischaligen Hyperboloide giebt es, wie auf dem Ellipsoide, zwei Schaaren Krümmungslinien, die von den confocalen Ellipsoiden und den einschaligen Hyperboloiden ausgeschnitten werden; die elliptischen Coordinaten eines Punktes ler Fläche sind somit nach den Gleichungen (1) $\sigma$ und $\mu$, während $\nu$ constant bleibt*).

Die von den einschaligen Hyperboloiden ausgeschnittenen Krümmungslinien durchziehen die ganze Flïche und können analytisch durch das Unendliche von einem Mantel auf den andern fortgesetzt werden, während die Krümmungslinien des andern Systems geschlossene Curven sind, die auf je einem Mantel verlaufen. Kür $\sigma=\mu=l$ erbält man den Axenschnitt $z=0$ (vergl. pag. 5059), d. h. derselbe gehört den Krümmungslinien beider Systeme an, während das eine System durch die Kreispunkte in das andere übergeht. Analytisch besteht also zwischen den Verhältnissen auf dem Hyperboloid und dem Ellipsoid bezüglich der Krümmungslinien kein Unterschied. Dasselbe gilt, wie man sich leicht überzeugt, von den geoditischen Linien. Die Differentialgleichung derselben ist:

wo:

$$
\frac{\left(\sigma^{2}-v^{2}\right) d\left(\sigma^{2}\right)}{S} \pm \frac{\left(\mu^{2}-\nu^{2}\right) d\left(\mu^{2}\right)}{M}=0
$$

$$
\begin{gathered}
S^{2}=\left(\sigma^{2}-k^{2}\right)\left(\sigma^{2}-\mu^{2}\right)\left(\sigma^{2}-h^{2}\right)\left(\sigma^{2}-\nu^{2}\right) \sigma^{2}, \\
M^{2}=\left(\mu^{2}-k^{2}\right)\left(\mu^{2}-\mu^{\prime 2}\right)\left(\mu^{2}-h^{2}\right)\left(\mu^{2}-\nu^{2}\right) \mu^{2} .
\end{gathered}
$$

Sie zeigt, dass jede geodätische Linie auf der einen Flächenhälfte nach zwei Richtungen hin in's Unendliche läuft. Einer der beiden $Z$ weige berührt seine zugehörige Krümmungslinie $\mu=\mu^{\prime}$ oder $\sigma=\mu^{\prime}$ auf derselben Flächenhälfte, wo er beginut, währeud der zweite durch's Unendliche fortgesetzt den andern Ast der Krürnmungslinie auf dem zweiten Flächenmantel tangirt. Betrachtet snan also die beiden Flächenmäntel als zusammengehörig, so oscilliren die Linien ebenso um die Fläche wie im Falle des Ellipsoides.

Da nuv $\mu^{\prime}=k$ werden kann, so wird es auch eine Linie geben, die die Krümmungslinien der beiden Systeme zugleich berabrt, was nư im Kreispunkte eintreten kann, und da ferner $\mu^{\prime}$ für alle Punkte der Geodätischen constant bleibt, so folgt, dass auch auf dieser Fläche die von einem Kreispunkte auslaufende Linie durch den entgegengesetaten Kreispunkt gehen muss. Daraus ist es aber evident, dass sich zwei unendlich benachbarte geodätische Linien, die von einem Kreispunkte

*) Durch Herro F. Klein wurde ich aufmerksam gemacht, dass es eine Collineation giebt, welche ein zweischaliges Hyperboloid und die geodatischen Linien auf ihm in ein Ellipsoid mit seinen geodätischen Linien verwandelt, woraus dann unmittelbar die Uebereinstimmung der folgenden Resultate mit deli für das Ellipsoid gewonnenen ersichtlich ist. 
auslaufen, niemals auf dem Flïchenmantel schneiden können, auf welchem der Ausgangspunkt liegt. Dieser Satz wurde von Herrn v. Mangoldt in dem bereits in der Einleitung erwähnten Aufsatze durch Transformation der Gleichung (24) nit elliptischen Functionen (was für $\mu^{\prime}=k$ möglich ist) allalytisch bewiesen*). Nach seiner Bezeichnung sind damn die Kreispunkte Punkte erster Art, während er unter Punkten zweiter Art solche versteht, durch welche zwei unendlich benachbarte geodïtische Linien gehen, die sich treffen, bevor sie das Unendliche erreichen.

\section{$\S 11$.}

\section{Grenzcurve anf dem Hyperboloid für den Bereich der Punkte, die zu Enveloppen Anlass geben.}

In meiner bercits mehrfach erwähnten Abhandlung habe ich darauf aufmerksam gemacht, dass beim zweischaligen Rotationshyperboloide der Fall eintreten kann, dass die ganze Enveloppe, die ja immer existirt, wenn man die beiden Flächentheile als zusammengehörig betrachtet, ganz auf dem zweiten Flächenmantel liegt, der den Büschelmittelpunkt der erzeugenden geodätischen Linien nicht enthält. Im gewöhnlichen Falle hingegen liegt die dem. Ausgangspunkt zugekehrte Spitze mit ihm auf demselben Mantel, während die andern drei sich auf der zweiten Flächenhälfte befinden. Der $Z$ wischenfall ist natürlich dann vorhanden, wenn die dem Büschelmittelpunkt zunächst gelegene Spitze gerade auf den unendlich fernen Kreis der Fläche füllt. Der Büschelmittelpunkt, der zu einer solchen Enveloppe Anlass giebt, wird dann wegen der Symmetrie der Fläche stets auf einem gewissen Parallelkreise $r_{0}$ liegen, für dessen Berechnung ich damals eine Integralgleichung aufstellte ${ }^{* * *}$ ).

Herr von Mangoldt fasst nun in der angefübrten Abhandlung nur einen Mantel der Fläche ins Auge und betrachtet nur diejenigen Schnittpunkte unendlich benachbarter geodätischer Linien, welche auf demselben Flächentheile mit dem Büschelmittelpunkte liegen. Deshalb ist für ihn der erwähnte Parallelkreis die Grenze, welche die Punkte erster und zweiter Art von einander trennt. Die Punkte erster Art liegen in dem den Scheitel der Fläche einschliessenden Theile, während die zweiter Art in dem äussern Raume liegen. Der Radius dieses Grenzkreises $r_{0}$ findet sich a. a. 0 . pag. 40 durch Einführung elliptischer Functionen berechnet, Die hierzu verwandte Gleichung unterscheidet sich. nicht wesentlich von der erwähnten Gleichung in meinem Aufsatze.

Nun überzeugt man sich durch ähnliche Betrachtungen wie beim dreiaxigen Ellipsoide leicht, dass der Verlauf der Enveloppe auf dem dreiaxigen Hyperboloide mit zwei Mantelflächen wenig verschieden ist

*) v. Mangoldt a. a. O. pag. 49-52.

- **) A. a. 0. pag. 564 . 
von dem Verfauf derselben auf dem Rotationshyperboloide, so dass also auch auf jener Flüche eine Grenzcurve für die Lage der Punkte erster und zweiter Art existiren wird*). Die ungefähre Gestalt dieser Curve bei verschiedenen Axenverhältnissen der Fläche wurde von Herrn v. Mangoldt angegeben. Es lässt sich aber auch, und zwar ausgehend von denselben geometrischen Betrachtungen, die ich früher für das Rotationshyperboloid angestellt, eine Gleichung zur Beștimmung - dieser Curve angeben.

Wie bei der Rotationsfläche, so erhält man auch hier die Punkte der Grenzcurve, wenn man fragt, welche Punkte als Büschelmittelpunkte geodütischer Linien genommen, Enveloppen erzeugen kömuen, deren dem Ausgangspunkte zun̈̈chst gelegene Spitze gerade auf der unendlich fernen Krümmungslinie der Fü̈che liegt. Diese Krümmungslinie gehört natürlich zum Systeme б. Die Spitze muss aber auch noch auf einer andern Krümmungslinie, nümlich auf $\mu_{0}^{\prime}$ liegen, wenn $\mu_{0}^{\prime}$ den Zweig von $\mu_{0}$ bedeutet, auf welchem der Ausgangspunkt nicht liegt. Denn $\mu_{0}$ wird ja im Büschelmittelpunkte von einer geodätischen Linie tangirt, deren Berührungspunkt mit $\mu_{0}{ }^{\prime}=\mu_{0}$ die Spitze festlegt (vergl. Ellipsoid § 6). Für alle Punkte der Grenzcurve liegt also $\mu_{0}$ stets zwischen $\mu=k$ und $\mu=h$.

Die Integralgleichung der geodätischen Linien ist dann für $\sigma_{0}$ und $\mu_{0}$ als Coordinaten des Ausgangspunktes:

$$
\int_{\sigma_{0}^{2}}^{\sigma^{2}} \frac{\left(\sigma^{2}-\nu^{2}\right) d\left(\nu^{2}\right)}{S}+\int_{\mu_{0}^{2}}^{\mu^{2}} \frac{\left(\mu^{2}-\nu^{2}\right) d\left(\mu^{2}\right)}{M}=0 .
$$

Da wir nur diejenige Linie ins Auge fassen, welche die $\mu_{0}$ im Ausgangspunkte beriuhrt, so haben wir statt $\mu^{\prime}$ in dieser Glejchung $\mu_{n}$ zu setzen, und da sich die auf $\mu_{0}^{\prime}=\mu_{0}$ gelegene Spitze im Unendlichen befinden soll, so muss für sie $\sigma=\infty$ sein; ihre Lage bestimmt sich somit aus der Gleichung

$$
\int_{\sigma_{0}^{2}}^{\infty} \frac{\left(\sigma^{2}-\nu^{2}\right) d\left(\nu^{2}\right)}{S_{0}}+2 \int_{\mu_{0}^{2}}^{h^{2}} \frac{\left(\mu^{2}-\nu^{2}\right) d\left(\nu^{2}\right)}{N_{0}}=0 .
$$

$S_{0}$ und $N_{0}$ sind die Werthe von $S$ und $N$ für $\mu^{\prime}=\mu_{0}$. Lässt man in dieser Gleichung $\mu_{0}$ alle Werthe von $k$ bis $h$ durchlaufen, so erhält man aus ihr jedesmal einen zuggehörigen $W$ erth $\sigma_{0}$, welcher mit $\mu_{0}$ einen Punkt der Grenzeurve festlegt.

Es sei jetzt

$$
\sigma^{2}=\mu^{2}=\frac{x\left(\mu_{0}^{2}-h^{2}\right) \nu^{2}-\left(\mu_{0}^{2}-\nu^{2}\right)}{x\left(\mu_{0}^{2}-h^{2}\right)-\left(\mu_{0}^{2}-\nu^{2}\right)}, \quad x^{2}=\frac{\mu_{0}^{2}-h^{2}}{\mu_{0}^{2}-\nu^{2}} \cdot \frac{k^{2}-\nu^{2}}{k^{2}-h^{2}},
$$




$$
\lambda^{2}=\frac{\mu_{0}^{2}-h^{2}}{\mu_{0}^{2}-\nu^{2}}, \quad \varrho^{2}=\frac{\mu_{0}^{2}-h^{2}}{\mu_{0}^{2}-\nu^{2}} \cdot \nu^{2} \cdot h^{2} .
$$

Dann geht $(25 \mathrm{~b})$ über in

$$
\int_{\frac{1}{\lambda^{2}}}^{x_{1}^{0}} \frac{d x_{1}}{\sqrt{X_{1}}}-2 \int_{1}^{0} \frac{d x_{2}}{\sqrt{X_{2}}}=0,
$$

wo $X=x(1-x)\left(1-x^{2} x\right)\left(1-\lambda^{2} x\right)\left(1-\varrho^{2} x\right)$ ist und $x_{1}^{0}$ dem Werthe $\sigma_{0}$ entspricht.

Um diese Gleichung nach $x_{1}{ }^{0}$ aufzulösen, betrachten wir, wie in $\S 1$, das Gleichungssystem:

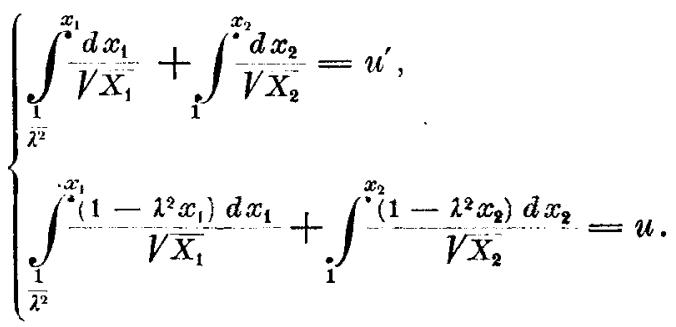

Transformirt man die $\vartheta$-Charakteristiken in den Gleichungen (8) $\S 2$ auf die Anfangswerthe $x_{1}=\frac{1}{\hat{\lambda}^{2}}, x_{2}=1$, so erhalt man aus den Gleichungen b), d) und e) folgende drei:

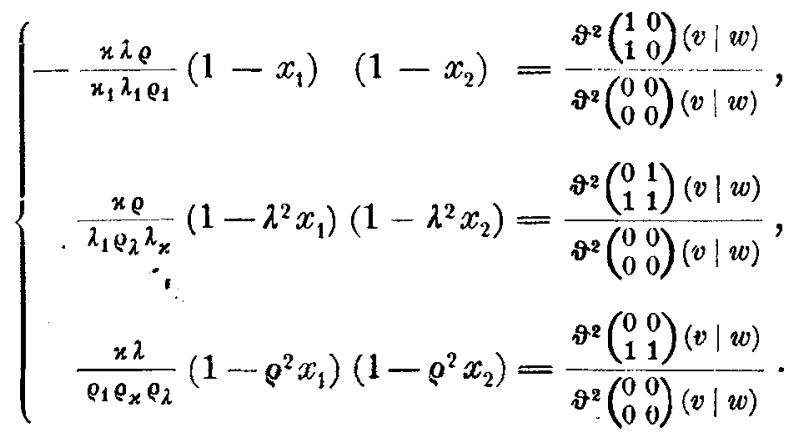

Macht man jetzt wieder die beiden unbeschränkt variablen Grössen $v$ und $w$ von $u$ und $u^{\prime}$ abhängig, indem man

$$
\begin{gathered}
v=\beta_{1,1} u+\beta_{1,2} u^{\prime}, \\
w=\beta_{2,1} u+\beta_{2,2} u^{\prime}
\end{gathered}
$$

setzt, und die $\beta$ anf dieselbe Art bestimmt, wie dies in $\S 3$ für das Ellipsoid geschehen, so sind, da $u^{\prime}$ constant, ja in unserm Falle sogar Null ist, $v$ und $w$ nur mehr von der einen Variabeln $u$ abhängig, und die obigen Formeln gehören den Punkten einer geodätischen Linie zu. 
Die geodätische Linie, welche wir ins Auge fassen, berührt im Unendlichen die Krümmungslinie $\mu_{0}^{\prime}$; für diesen Punkt ist $x_{1}=\frac{1}{\lambda^{2}}$, $x_{2}=1$, somit $u=0$, und aus der ersten Formel $(27)$ folgt $\vartheta\left(\begin{array}{l}10 \\ 10\end{array}\right)(v \mid w)=0$. Diese $\vartheta$-Function verschwindet aber nicht bloss für $u=0$, sondern so of $\mu=\mu_{0}$ wird. Denkt man sich also jetzt umgekehrt die Linie im unendlich fernen Berührungspunkte mit $\mu_{0}^{\prime}\left(x_{1}=\frac{1}{\lambda^{2}}, x_{2}=1\right)$ beginnend, so wird $\vartheta\left(\begin{array}{l}10 \\ 10\end{array}\right)(v \mid w)$ alsbald verschwinden, wenn die geodätische Linie den andern $Z$ weig $\mu_{0}$ der Krümmungslinie tangirt, d. h. in dem gesuchten Punkte der Grenzcurve.

Bestimmt man also aus der Glcichung

$$
\vartheta\left(\begin{array}{ll}
1 & 0 \\
1 & 0
\end{array}\right)(v \mid w)=0
$$

die Wurzel $u_{0}$, so legt diese den Schnittpunlt der Krïmmungslinie $\mu_{0}$ mit unserer Grenzcurve fest.

Ferner folgt aus der oben gebrauchten Substitution und der dritten und zweiten der Gleichungen (27)

$$
\sigma=\nu \sqrt{\frac{h}{\mu_{0}}} \sqrt[4]{\frac{k^{2}-v^{2}}{h^{2}-\nu^{2}}} \cdot \frac{\vartheta\left(\begin{array}{ll}
0 & 0 \\
1 & 1
\end{array}\right)(v \mid w)}{\vartheta\left(\begin{array}{ll}
0 & 1 \\
1 & 1
\end{array}\right)(v \mid w)},
$$

und $u_{0}$ in diese Gleichung eingetührt, liefert den verlangten Parameter $\sigma_{0}$, der mit $\mu_{0}$ einen Punkt der Grenzcurve festlegt. Natürlich wäre es auch leicht, die rechtwinkligen Coordinaten dieses Purktes als Functionen von $u$ darzustellen.

Durch die Gleichungen (28) und (29) ist somit die Grenzeurve vollkommen bestimmat.

München im Juli 1882. 